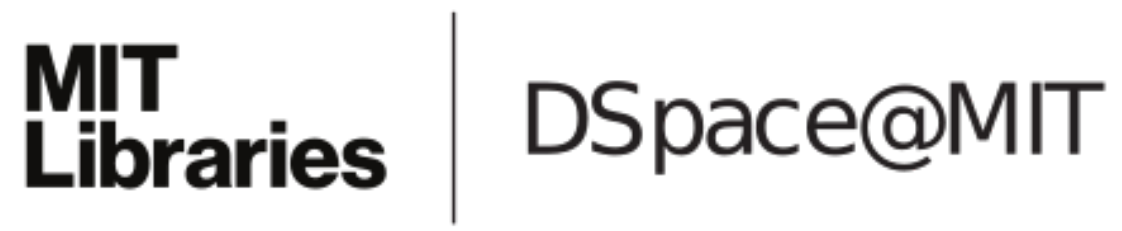

\author{
MIT Open Access Articles
}

Describing the firmness, springiness and rubberiness of food gels using fractional calculus. Part II: Measurements on semi-hard cheese

The MIT Faculty has made this article openly available. Please share how this access benefits you. Your story matters.

Citation: Faber, T.J. et al. "Describing the Firmness, Springiness and Rubberiness of Food Gels Using Fractional Calculus. Part II: Measurements on Semi-Hard Cheese." Food Hydrocolloids 62 (January 2017): 325-339 ๔ 2016 Elsevier Ltd

As Published: http://dx.doi.org/10.1016/J.FOODHYD.2016.06.038

Publisher: Elsevier

Persistent URL: http://hdl.handle.net/1721.1/113213

Version: Author's final manuscript: final author's manuscript post peer review, without publisher's formatting or copy editing

Terms of use: Creative Commons Attribution-NonCommercial-NoDerivs License 


\title{
Describing the firmness, springiness and rubberiness of food gels using fractional calculus. Part II: Measurements on semi-hard cheese.
}

\author{
T.J. Faber ${ }^{\mathrm{a}, \mathrm{b}, 1, *}$, A. Jaishankar' ${ }^{\mathrm{c}}$, G.H. McKinley ${ }^{\mathrm{c}}$ \\ ${ }^{a}$ FrieslandCampina, PO box 1551, 3800 BN Amersfoort, The Netherlands \\ ${ }^{b}$ Polymer Technology, Eindhoven University of Technology, PO Box 513,5600 MB Eindhoven, The \\ Netherlands \\ ${ }^{c}$ Department of Mechanical Engineering, Massachusetts Institute of Technology, \\ 77 Massachusetts Avenue, Cambridge - MA 02139, USA
}

\begin{abstract}
We use the framework of fractional calculus to quantify the linear viscoelastic properties of full-fat, low-fat, and zero-fat, semi-hard cheeses over a range of temperatures and water / protein ratios. These fractional constitutive models correctly predict the time-dependence and interrelation of the firmness, springiness, and rubberiness of these emulsion-filled hydrocolloidal gels. Our equations for the firmness $F$, springiness $S$, and rubberiness $R$, also correctly predict the effect of changing the magnitude or time-scale of the stress loading on the material even in the case of irreversible flow events, when cheese progressively transitions from a solid to a liquid. Finally we show how our FSR-equations can be used in a texture engineering context; they provide rational guidance to product reformulation studies and allow for extrapolation of a firmness measurement to practical situations in which the gel is subjected to prolonged creep loading.
\end{abstract}

Keywords: rational reformulation, food gels structure-texture engineering, constitutive model, fractional calculus, Scott Blair

\footnotetext{
v 5

*corresponding author

Email address: timo.faber@frieslandcampina.com (T.J. Faber)
} 


\section{Introduction}

Cheese is one of the most researched products in terms of rheology-texture relationships (Davis, 1937; Scott Blair and Coppen, 1942b; Luyten, 1988; Walstra and van Vliet, 1991; Gunasekaran and Ak, 2003; Foegeding and Drake, 2007). It is the canonical example of a food gel for which having the right level of firmness is of pivotal importance. The firmness of cheese demarcates the different types of cheese on physical grounds (Davis, 1965, 1937) and groups them into soft, semi-soft, semi-hard and hard varieties (Gunasekaran and Ak, 2003). The level of firmness determines whether we use utensils or hands when consuming a cheese. When offered a type of cheese, e.g. Cheddar, Gouda or Parmesan cheese, consumers have specific expectations regarding its firmness (Yates and Drake, 2007). Finally, having the right firmness is not only important to consumers, it is as critical for efficient production of cheese as well. All handling from whey drainage to portioning, storing and slicing (Scott Blair and Coppen, 1940a; Johnson and Law, 2010) is adapted to the cheese firmness.

Shortly after the field of rheology was founded, Davis (1937) and Scott Blair (Scott Blair et al. 1947) were among the first to apply concepts from this new area of science to find the essential properties that determine cheese texture. Davis' incentive was to develop proper instrumental measures for firmness and springiness for quality control. He constructed a simple compression apparatus from Meccano parts and performed creep / recovery tests in compression to determine an apparent shear modulus $G$ and viscosity $\eta$. In a table he showed that the firmness of cheese, as graded by professional graders, were placed in the same sequence as the measured magnitudes of modulus and viscosity. He suggested that the springiness $S$ is quantified as a Maxwellian relaxation time $S^{(\text {Davis })} \equiv \tau_{r}=\eta / G$. To our knowledge this is the first equation expressing a texture attribute in terms of essential material properties.

Davis (1937) described the creep phase of his experiment as a period of flow and elastic compression. At the end of the recovery period, he assumed an equilibrium state and measured the elastic recoverable deformation $e$, and plastic non-recoverable deformation, which he denoted as plastic flow $f$. He pointed out the importance of discriminating between severe and mild load cases since it could greatly affect the response of the cheese. He introduced 
the term "stress-time" to indicate the severity of the loading; a combination of the weight put on the cheese plug and the time taken to follow the response in both the creep and recovery phase. He did not quantify stress-time but only used it in qualitative sense, speaking of high and low stress-time conditions or periods. Since the introduction of Texture Profile Analysis (TPA) in food research (Szczesniak, 1963; Friedman et al., 1963) the uniaxial compression experiment has become more popular over the creep / recovery experiment to measure food texture. In the uniaxial compression experiment, loading is typically applied by imposing a constant rate of axial displacement or compressive strain on the material. Recovery from the loading is measured by reversing the direction of the displacement.

By contrast, in the creep / recovery experiment, loading is applied by imposing a constant stress over a defined period of time, and the unloading is achieved by setting the stress to zero and measuring the recoil in the sample. We favour the latter experiment to determine the firmness, springiness, and rubberiness since this protocol more closely resembles the loading experienced by the material in actual use conditions and allows us to connect the firmness to situations where stresses are applied for short times, such as sensory texture measurement, or for long times, such as in storing cheese. When performed in a modern torsional shear rheometer we can compare and contrast the firmness, springiness, and rubberiness of food gels spanning a wide variety of modulus or viscosity, whereas the uniaxial compression experiment requires the gel to be able to hold its own weight.

Goh et al. (2003) show that the power-law relaxation characteristics of hard and semihard cheese can be characterized in compression by fitting a constitutive equation of the form $\sigma=\phi \varepsilon^{m}\left(t / t_{r}\right)^{-n}$ to uniaxial monotonic compression data. Here $\sigma$ is the stress difference in the material, $\phi$ is a pre-factor with units of $\mathrm{Pa}, m$ and $n$ are power-law exponents for the strain $\varepsilon$ and time $t$ respectively and $t_{r}$ is the arbitrarily chosen reference time of $1 \mathrm{~s}$. They argue that the pre-factor $\phi$ is more suitable for comparing the firmness of various food materials, because in contrast to the quasi-property $\Psi$ (Faber et al., 2015), it does not involve a fractional power of time. As we describe later, we choose to retain the time-dependency in our definition of firmness however, because this allows us to extrapolate the measured firmness to how a food gel performs under practical conditions, e.g. whether it will retain shape when stored on a 
shelf.

Cheese is a beautiful example of a textured food having a multi-scale and self-similar structure as depicted by the sequence of images shown in Fig. 1. On the scale of $1-100$ $\mu \mathrm{m}$, cheese is a filled gel (Fig. 1(d)) (Luyten, 1988; Yang et al., 2011) of fat globules with characteristic size 1-3 $\mu \mathrm{m}$ (Walstra, 1968) (Fig. 1(c)) dispersed in a gel of protein and water (Fig. 1(b)). At temperatures below $15^{\circ} \mathrm{C}$, the filler is stiff and elastic and cheese is more accurately described as a 'suspension-filled gel'. At higher temperatures, the filler becomes viscoelastic and we may instead consider an 'emulsion-filled gel' (Dickinson, 2012). In milk, casein is arranged in hydrocolloidal clusters with a diameter of approximately $200 \mathrm{~nm}$, often denoted as casein micelles (De Kruif et al., 2012). When adding rennet together with calcium to milk at a temperature of $30^{\circ} \mathrm{C}$, the steric layer ( $\kappa$-casein) present around the casein micelle is cut off and fractal aggregates or flocks of attractive para-casein colloids are formed, which end up forming a percolating structure. The gel is further concentrated through a process called syneresis (Van Vliet and Walstra, 1994), in which water and water-soluble materials are expelled from the network. The end result is a cheese that contains roughly equal amounts of fat, protein and water and a liquid by-product called whey.

In the present study (which is part II of a brace of papers) we show that irrespective of fat, protein or water content, in the linear viscoelastic regime our cheese displays powerlaw relaxation over a wide range of frequencies and we explain how to evaluate the quasiproperties and exponents that uniquely characterize each material from measurements of the storage and loss modulus $\left\{G^{\prime}(\omega), G^{\prime \prime}(\omega)\right\}$. We show that from these parameter values we can correctly predict the evolution of both the relaxation modulus $G(t)$ and the creep compliance $J(t)$. In part I of this work we have introduced exact definitions of the textural attributes of firmness $F$, springiness $S$, and rubberiness $R$, for food gels in terms of specific points on the creep / recovery curve. This allows us to derive analytical expressions for the material firmness, springiness, and rubberiness in terms of the quasi-property and the power-law exponent that characterize each cheese. We show that we can predict the springiness and rubberiness of the sample from the creep compliance curve and demonstrate that firmness, springiness, and rubberiness are interrelated through the fractional constitutive equations in 


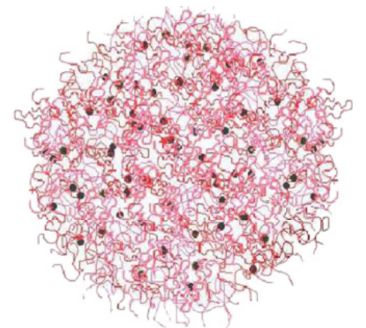

(a) hydro colloid

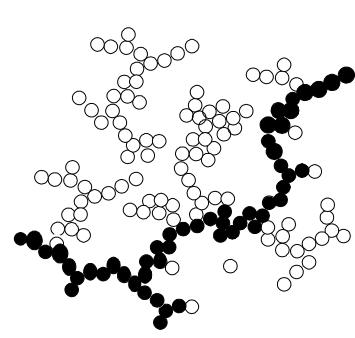

(b) fractal strands

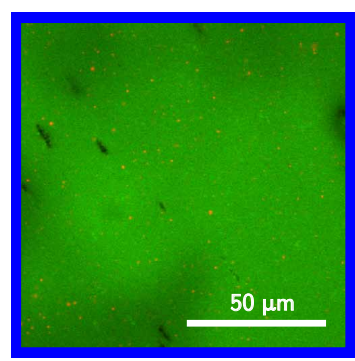

(c) gel

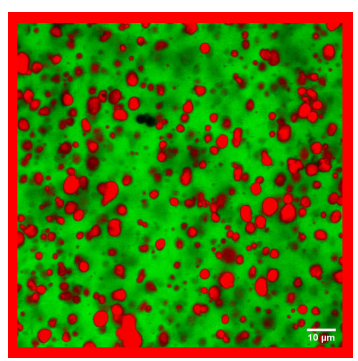

(d) filled gel

Figure 1: Cheese as a complex multi-scale material. Casein, the main protein in cheese forms a hydrocolloid in milk. An artist impression of this hydrocolloid, often denoted as the casein micelle and abbreviated as $\mathrm{CN}$, is depicted in (a) (colored image, taken from De Kruif et al. (2012), artist impression originally published in De Kruif and Holt (2003), reprinted with permission). The casein micelle has a diameter $d_{C N} \approx 200 \mathrm{~nm}$ (De Kruif et al. 2012) and contains colloidal Calcium-Phosphate (CCP) nano-clusters with size $d_{C C P} \approx 4 \mathrm{~nm}$ (De Kruif et al. 2012), depicted as black dots in Fig. (a). Around these nano-clusters the concentration of protein is more dense. In cheese production the interfacial steric layer of $\kappa$-casein (with size $d_{\kappa-C N} \approx 7 \mathrm{~nm}$, De Kruif et al. (2012)) that is present around the casein micelle is cut off and attractive para-casein micelles (p-CN) are formed. The latter colloids aggregate into (b) a fractal, space-spanning, structure (Bremer et al., 1990) to form a water-holding gel, with a stress-carrying backbone (in black, picture taken from Kloek (1998) with author's permission). After removal of whey, a concentrated gel with a water-protein ratio $w / p=1.8$ remains as depicted in the micro graph in (c), obtained by Confocal Scanning Light Microscopy (CSLM). Material properties of the unfilled gel are obtained by measuring the rheological response of zero-fat cheese produced from skimmed milk; the corresponding rheological data is color-coded blue throughout this paper. (d) If cheese is produced from milk that contains fat, the suspended fat globules present in the milk are occluded by the protein network, and an emulsion-filled gel is obtained, as depicted in the CSLM image in (d). In full-fat cheese, the fat volume fraction is typically $\Phi_{f}=30 \mathrm{v} / v \%$. Material properties of the filled gel are obtained by measuring the rheology of full-fat cheese, and the corresponding rheological data is color-coded red throughout this paper.

terms of just two material parameters that we can determine in the linear viscoelastic regime. We finally demonstrate that our equations give correct, quantitative predictions of the effect of stress-time loading on the values of the textural attributes of $F, S$ and $R$. Subsequently we combine model fits from 40 combinations of cheese composition and temperature into a firmness, springiness, and rubberiness master plot. This shows us at a glance that the operating window for cheese reformulation is limited and that novel, firmness-enhancing structures are required. In the discussion section we outline how our equations can be used in the context of structure-texture design. 


\section{Materials and methods}

\subsection{Cheese composition}

Foil-ripened Gouda rectangular cheeses $(500 \times 300 \times 100 \mathrm{~mm})$ were acquired at an age of 3-14 days and kept at $5^{\circ} \mathrm{C}$ to minimise compositional changes due to protein breakdown or (de-)solubilization of minerals (Lucey et al., 2005; O'Mahony et al., 2006) . Fat content was varied by using cheese from three fat classes: zero-fat $(\approx 0 \%$ fat in dry matter, fidm), low fat $(\approx 20 \%$ fidm $)$ and full-fat $(\approx 48 \%$ fidm $)$. The cheese was analyzed for composition according to international standards (standard in brackets): $\mathrm{pH}$ (NEN 3775, Netherlands Normalisation Institute), 1-lactic acid (ISO 8069, International Standard Organisation), protein (through total nitrogen / soluble nitrogen / anhydrous nitrogen fractions (Visser, 1977)), ash (Association of Official Analytical Chemists 930.30), calcium (insoluble calcium phosphate, AOAC 984.27), lactose (ISO 5762-2), water (=100-total solids (ISO 5534)), fat (ISO 1735) and chloride (ISO 5943). Weight fractions of protein, water and fat were converted to volume fractions according to the procedure outlined by Yang et al. (2011) taking the temperature-dependent densities of these main cheese constituents from Sahin and Sumnu (2006).

\subsection{Cheese hydration}

Cheese slices of approximately $60 \times 60 \times 2.5 \mathrm{~mm}$ were cut from a block coming from the core of the cheese. To provide cheese with different water/protein ratios (denoted as $w / p$ ), the hydration procedure developed by Luyten (1988) was followed, with slight adaptations for shear rheometry. Part of the slices were hydrated in a salt solution, which had equal concentration of calcium $\left(\mathrm{Ca}^{2+}\right)$ and chloride $\left(\mathrm{Cl}^{-}\right)$as in the moisture of the non-hydrated cheeses on a molar basis. For the fraction of soluble calcium (normalized with calcium), a value of 20\% was assumed (McMahon et al. 2005). Hydration was performed by submersing a single cheese slice for $1,2,4,8,16$ or 24 hours in $250 \mathrm{ml}$ of the salt solution. After this period, slices were taken from the liquid and excess moisture was carefully removed with tissue paper. Just before and after hydration the slices were weighed. From the weight increase the new water/protein ratio was calculated, assuming that the concentration of solubles in the cheese moisture remained the same and that there was no net transfer of material from cheese 
to the immersion liquid. Slices were wrapped in aluminum foil and kept in the refrigerator for 2-3 days to allow for moisture equilibration (Luyten, 1988).

\subsection{Small strain shear rheology}

Experiments were performed at $10^{\circ} \mathrm{C}, 25^{\circ} \mathrm{C}$ and $30^{\circ} \mathrm{C}$. From each cheese slice, three discs of $25 \mathrm{~mm}$ diameter were punched for parallel-plate rheometry. When measurements from frequency sweeps were compared against stress relaxation or creep experiments, samples were taken from the same slice. Measurements were performed with a Physica MCR501 Rheometer (Anton Paar, Austria) with a parallel-plate geometry. To prevent slip, sandblasted upper and lower plates are used. The temperature of the lower plate was controlled with a Peltier stage, and the upper plate and cheese environment were thermally controlled with a cap hood. The upper plate was lowered with a speed of $25 \mu \mathrm{m} / \mathrm{min}$ until a normal force of $1 \mathrm{~N}$ (corresponding to a normal stress of $4 \mathrm{kPa}$ ) was reached. The gap width was recorded at that point and decreased by an extra $2 \%$ while keeping the normal force constant at $1 \mathrm{~N}$ to ensure full contact with the cheese. Gap settings were then switched from fixed normal force to fixed gap width. No significant effect of normal pressure on storage and loss modulus was found in the range of $0.5-20 \mathrm{kPa}$. After loading the sample between the two parallel plates it was heated at a heating rate of $0.5^{\circ} \mathrm{C}$ per minute until the desired temperature was reached. The exposed surface area of the sample was covered with sunflower oil to minimise sample drying during the experiment. A maximum weight loss of $0.5 \mathrm{w} / \mathrm{w} \%$ was recorded.

Linear viscoelastic region (LVR). To determine the LVR a strain sweep at a frequency of 1 $\mathrm{rad} / \mathrm{s}$ was conducted with a logarithmic increase of the strain amplitude $\gamma_{0}$ from 0.1 to $100 \%$. Strain sweeps were performed at temperatures of $T=10^{\circ} \mathrm{C}$ and $T=25^{\circ} \mathrm{C}$.

Storage and Loss Modulus $\left\{G^{\prime}(\omega), G^{\prime \prime}(\omega)\right\}$. Frequency sweeps were performed at a strain amplitude $\gamma_{0}=0.2 \%$, which lies within the LVR for all samples. The frequency was decreased logarithmically from $\omega=100 \mathrm{~Hz}$ to $\omega=0.1 \mathrm{~Hz}$ at fixed measuring temperatures of either $T=10^{\circ} \mathrm{C}$ and $T=30^{\circ} \mathrm{C}$. The heating rate inbetween the two sweeps was $0.5^{\circ} \mathrm{C}$ per minute. 
Relaxation modulus $(G(t))$. A step-strain of $\gamma_{0}=0.2 \%$ was imposed on the test specimen and held at that value for $t=100 \mathrm{~s}$ at a measuring temperature of $T=10^{\circ} \mathrm{C}$.

Creep compliance $(J(t))$. A step stress of $\sigma_{0}=100$ or $1000 \mathrm{~Pa}$ was imposed on the test specimen and held at this value for $t_{f}=10 \mathrm{~s}$ or $t_{f}=100 \mathrm{~s}$ at fixed measuring temperatures of either $T=10^{\circ} \mathrm{C}$ or $T=25^{\circ} \mathrm{C}$, while measuring the evolution in the resulting strain $\gamma(t)$. Subsequently the imposed stress is released and the resulting strain recovery or recoil is measured for $t_{r}=10 \mathrm{~s}$ or $t_{r}=100 \mathrm{~s}$.

\subsection{Confocal Scanning Laser Microscopy (CSLM)}

A Leica inverted CSLM (TCS SP2, DM IRE2) was used in the experiments. The water/protein phase was stained with fluoroscein isothiocyanate (FITC) and the fat phase with Nile red $(0.1 \% / 0.01 \%)$. Staining occurred by placing a sample of approximately $1 \times 5 \times 5 \mathrm{~mm}$ in a solution of the dyes in a glycerol / water / polyethyleneglycol (PEG) (45/5/50\%) mixture for 30 minutes. All cheese manipulations (cutting and staining) were done at $8^{\circ} \mathrm{C}$ in the cold room to prevent fat melting. Stained cheese was transported to the confocal microscope in a Petri dish placed in a polystyrene foam box containing a frozen ice pack isolated by rubber foam. Image acquisition was done below $15^{\circ} \mathrm{C}$ using a conditioned air flow. Single 2D images were obtained from the internal structure, imaging at about $10 \mu \mathrm{m}$ below the surface generated with a razor blade. The size frame of all images was $119.05 \times 119.05 \mu \mathrm{m}(1024 \times 1024$ pixels) obtained with a water immersion objective $(63 \times$, zoom 2 , NA $=1.2)$. Baseline adjustment and auto-dye-finding operations were applied to all images acquired using LEICA Confocal Software (LCS). 


\section{Results}

\subsection{Determining constitutive parameters for cheese}

In Fig. 2 we display a subset of the 40 frequency sweeps that were performed on Gouda cheese samples varying in temperature and composition. This subset consists of full-fat and zero-fat cheese at temperatures $T=10^{\circ} \mathrm{C}$ and $T=30^{\circ} \mathrm{C}$ and a water/protein ratio of $w / p=$ 1.8. In all cases the linear viscoelastic properties of the cheese show the typical power-law behaviour of a critical gel (Winter and Mours, 1997): i.e. a line of constant slope for both the storage and loss modulus, $\left\{G^{\prime}(\omega), G^{\prime \prime}(\omega)\right\}$ on a log-log plot over a wide range of frequencies, with both curves nearly parallel. To retrieve the quasi-properties and exponents that characterize each material from these plots, we need analytic expressions for the storage and loss modulus predicted by the Scott Blair model (see Eq. (1) of Faber et al. (2015): for convenience we have also summarized the key equations from part I in the Appendix; Table A.1. The complex modulus is obtained by Fourier transforming the constitutive equation for the springpot, Eq. A.1, which results in:

$$
G^{*}(\omega)=\mathbb{G}(i \omega)^{\beta}
$$

Following the procedure for separating out the real and the imaginary part, outlined by Friedrich et al. (1999) and Schiessel et al.(1995), one can readily find for the storage modulus

$$
G^{\prime}(\omega)=\mathbb{G} \omega^{\beta} \cos (\pi \beta / 2)
$$

and for the loss modulus

$$
G^{\prime \prime}(\omega)=\mathbb{G} \omega^{\beta} \sin (\pi \beta / 2)
$$

The magnitude of the complex modulus, $\left|G^{*}(\omega)\right|$, can be calculated from

$$
\left|G^{*}(\omega)\right|=\sqrt{\left(\mathbb{G} \omega^{\beta} \cos (\pi \beta / 2)\right)^{2}+\left(\mathbb{G} \omega^{\beta} \sin (\pi \beta / 2)\right)^{2}}=\mathbb{G} \omega^{\beta} .
$$




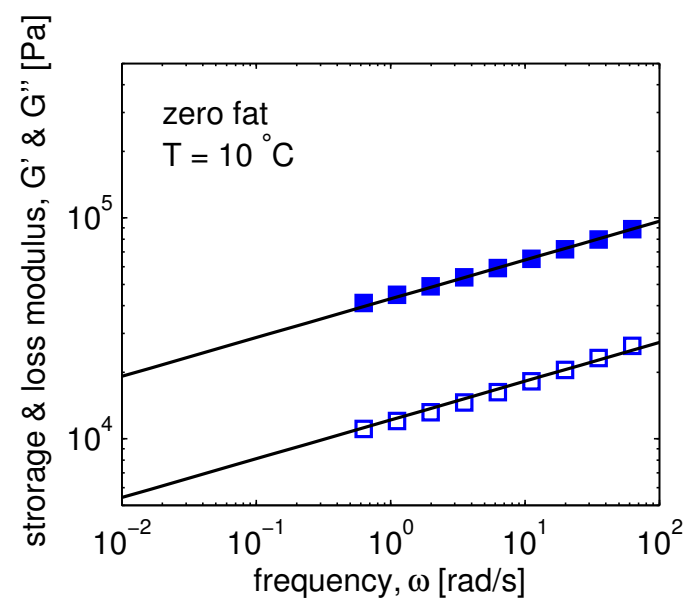

(a)

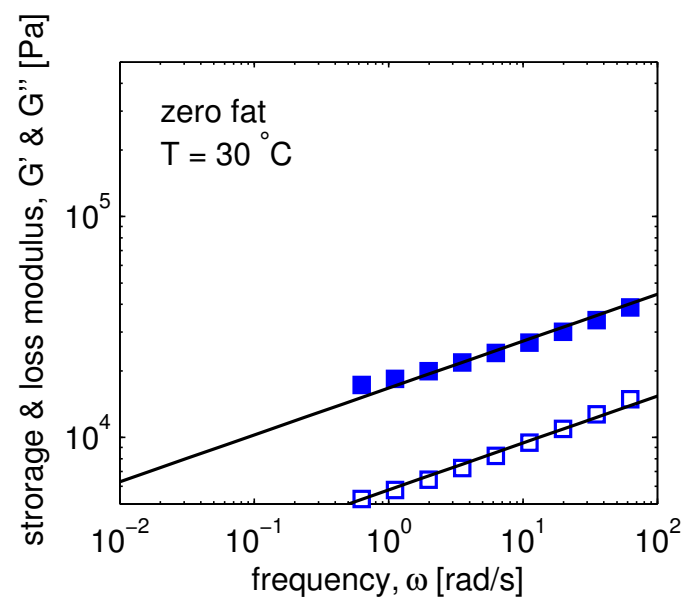

(c)

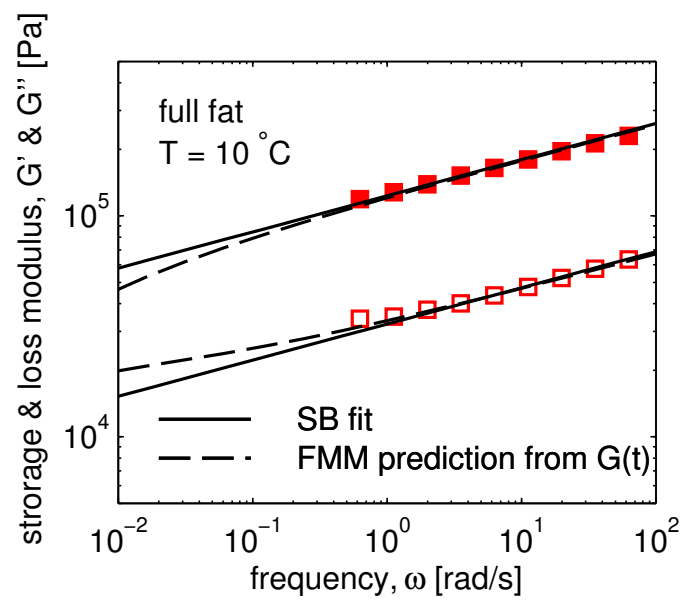

(b)

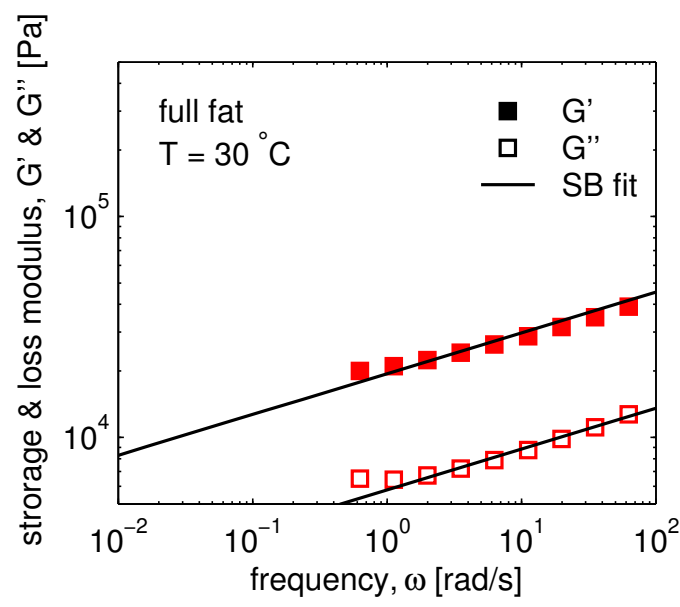

(d)

Figure 2: Determining the quasi-properties and fractional exponents of zero-fat $(\mathbf{a}, \mathbf{c})$ and full-fat $(\mathbf{b}, \mathbf{d})$ cheese with a water/protein ratio $w / p=1.8$, at temperatures $T=10^{\circ} \mathrm{C}(\mathbf{a}, \mathbf{b})$ and $T=30^{\circ} \mathrm{C}(\mathbf{c}, \mathbf{d})$. The material parameters are obtained by fitting the Scott Blair model (SB) for the complex modulus, Eq. (4) to the storage and loss modulus measurements $\left\{G^{\prime}(\omega), G^{\prime \prime}(\omega)\right\}$. The SB model gives a good fit for all samples, demonstrating that the relaxation behaviour of cheese is well described by a single powerlaw over frequencies $1 \leq \omega \leq 100$ $\mathrm{rad} \mathrm{s}^{-1}$. The additional dashed lines shown in Fig. $2(\mathbf{b})$ are low-frequency predictions from an independent measurement of the relaxation modulus, $G(t)$, of the same material (measurement displayed in Fig. 33(a)), over an extended range of timescales $0.01 \leq t \leq 200 \mathrm{~s}$. 
This set of equations shows that $G^{\prime}(\omega)$ can be predicted from $G^{\prime \prime}(\omega)$ and vice versa and that we can either fit equations (2), (3) or (4) to our dataset of $G^{\prime}(\omega), G^{\prime \prime}(\omega)$, or $\left|G^{*}(\omega)\right|$ respectively. We choose Eq. (4) in combination with a least square optimization procedure to obtain $\mathbb{G}$ and $\beta$, since it gives the least bias towards either the $G^{\prime}(\omega)$ or $G^{\prime \prime}(\omega)$ data points. The reconstituted curves for the storage and loss moduli predicted by the SB model are depicted by the solid lines in Fig. 2, and show that the model gives a good fit for both moduli, with only two material parameters. The values for the model parameters can be found in Table A.2. The fractional exponent $\beta$ varies between 0.16 and 0.21 depending on temperature and fat content. These values are in line with what Goh et al. (2003) found for Gouda cheese and Zhou and Mulvaney (1998) found for their model cheese gels. In the introduction we noted that the tangent of the phase angle $\tan (\delta)$, is commonly employed for gelled foods to express their solid- or liquid-like nature (Foegeding et al. 2011). For the SB model the phase angle is independent of the frequency and is only a function of the exponent $\beta$ :

$$
\tan (\delta)=\frac{G^{\prime \prime}(\omega)}{G^{\prime}(\omega)}=\tan (\pi \beta / 2)
$$

Thus our cheese is more elastic than viscous in character (or equivalently more solid- than liquid-like).

In Fig. 3 we use the quasi-property, $\mathbb{G}$, and fractional exponent, $\beta$, obtained from frequency sweeps, to predict a priori the relaxation modulus $(G(t)$, Fig. $3(\mathbf{a}))$ and creep compliance $(J(t)$, Fig. $3(\mathbf{b}))$ of zero-fat and full-fat cheese. We compare the predictions against direct measurements of these linear viscoelastic material functions. Fig. $3(\mathbf{b})$ shows that at $t \approx 10^{-2} \mathrm{~s}$ creep measurements for both cheese first superpose and then exhibit highly damped oscillations until the ultimate power-law regime is reached at $t \approx 10^{-1} \mathrm{~s}$. This characteristic short time response is due to a coupling between the complex modulus of the material with the moment of inertia of the rheometer and is often referred to as 'creep ringing'. It has been extensively studied by Jaishankar and McKinley (2013) for the Fractional Maxwell Model, resulting in a higher-order fractional differential equation that predicts the damped response of power-law materials in the ringing regime at short times as well as at longer times (for 


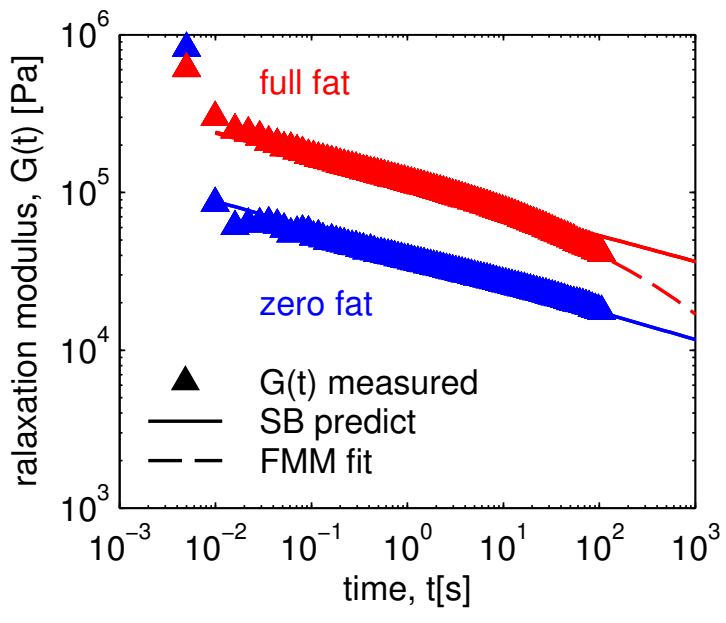

(a)

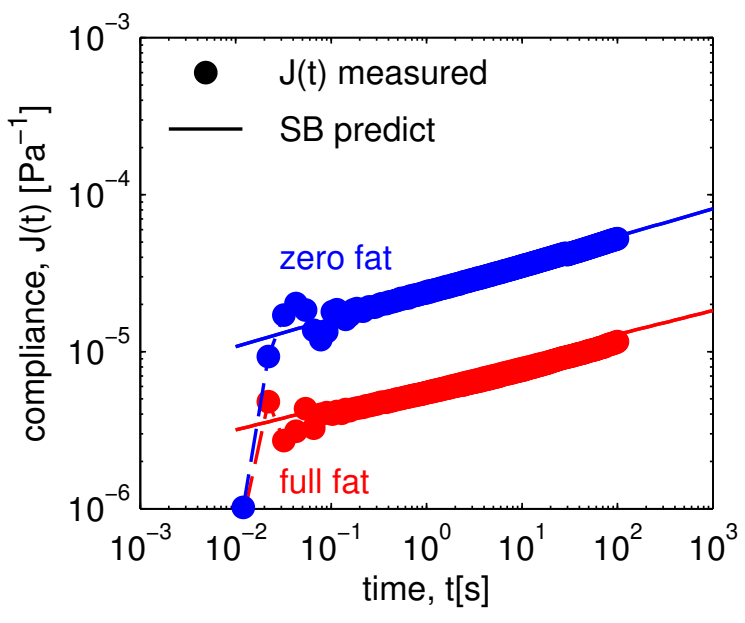

(b)

Figure 3: Measurement and prediction of (a) relaxation modulus $G(t)$ and (b) creep Compliance $J(t)$ of zero-fat (blue) and full-fat (red) cheese at a temperature $T=10^{\circ} \mathrm{C}$ and for a water/protein ratio $w / p=1.8$. (a,b) Zero-fat cheese shows single power-law behavior up to times $t=100 \mathrm{~s}$ of relaxation or creep. (a) Full-fat cheese starts to deviate from a single power-law response after times $t=10 \mathrm{~s}$ of stress relaxation. The second power-law relaxation process is captured with a fit of the Fractional Maxwell Model of the relaxation modulus (dashed line).

brevity a corresponding analysis is not included here). Similar 'ringing' for the measurements of the relaxation modulus $G(t)$ at times $t<10^{-1} \mathrm{~s}$, can be seen in Fig. 3 (a). Additional contributions to the high values of $G(t)$ measured at times $t<10^{-1} \mathrm{~s}$, are the Rouse modes of the biopolymer network ( $\mathrm{Ng}$ and McKinley, 2008). However this should give an initial slope of $d(\log (G(t))) / d(\log (t))=-0.5$ (Bagley and Torvik, 1983), which is not the case here.

To predict the relaxation modulus from the material parameters $\mathbb{G}$ and $\beta$, we need an analytical expression for $G(t)$. This is obtained by substituting the step-strain deformation in the constitutive equation for the springpot (Eq. (A.1)) and taking the Laplace transform to solve for $G(t)=\sigma(t) / \gamma_{0}$. Inverse transforming the result gives (Jaishankar and McKinley, 2013):

$$
G(t)=\frac{\mathbb{G} t^{-\beta}}{\Gamma(1-\beta)}
$$

where $\Gamma(z)$ is the Gamma function (Abramowitz and Stegun, 1964). The predictions of this two-parameter Scott Blair model (SB) are plotted as solid lines in Fig. $3(\mathbf{a})$. We see that for zero-fat cheese, both the magnitude and the slope of $G(t)$ are correctly predicted by Eq. (6). 
For full-fat cheese, the SB model (fitted to frequency data over the range $\omega \geq 0.5 \mathrm{rad} \mathrm{s}^{-1}$ ) gives good predictions at times $t \lesssim 2 \pi / \omega_{\min }=12 \mathrm{~s}$. For times $t \gtrsim 12 \mathrm{~s}$, a second powerlaw relaxation process with a steeper slope dominates the dynamics. This can be quantified by adding an additional springpot in series with the first Scott Blair element, which leads to the constitutive equation for the Fractional Maxwell Model (or FMM,Eq. (A.2)). Following the same procedure as that followed for constructing the Scott Blair model, we arrive at an expression for the relaxation modulus of the FMM (Friedrich et al., 1999; Jaishankar and McKinley, 2013):

$$
G(t)=\mathbb{G} t^{-\beta} E_{\alpha-\beta, 1-\beta}\left(-\frac{\mathbb{G}}{\mathbb{V}} t^{\alpha-\beta}\right)
$$

where $E_{a, b}(z)$ is the generalized Mittag-Leffler function defined as (Podlubny, 1999)

$$
E_{a, b}(z)=\sum_{k=0}^{\infty} \frac{z^{k}}{\Gamma(a k+b)}, \quad(a>0, b>0)
$$

By using the definition for the characteristic time constant in Eq. (A.13), the (dimensionless) argument of the Mittag-Leffler function in Eq. (7) is recognized to be $z=-\left(t / \tau_{c}\right)^{\alpha-\beta}$. where $\tau_{c}$ is the characteristic time-scale of the Fractional Maxwell model (Eq. (A.13), Faber et al. (2015)). When we fit Eq. (7) to the full-fat cheese measurements shown in Fig. 3(a), we obtain the dashed line. The second Scott Blair element has a fractional exponent $\alpha=0.65$ and is more viscous in character than the first element, which has an exponent $\beta=0.14$. We can check whether the obtained fit values represent true material properties by seeing how they independently predict the storage and loss modulus $\left\{G^{\prime}(\omega), G^{\prime \prime}(\omega)\right\}$ of the same cheese material, by substituting the four model parameters $\{\mathbb{G}, \mathbb{V}, \alpha, \beta\}$ into the expression for $G^{\prime}(\omega)$ and $G^{\prime \prime}(\omega)$ predicted by the Fractional Maxwell model. This model is obtained by taking the Fourier transform of Eq. (A.2) which gives (Jaishankar and McKinley, 2013):

$$
G^{*}(\omega)=\frac{\mathbb{V}(i \omega)^{\alpha} \cdot \mathbb{G}(i \omega)^{\beta}}{\mathbb{G}(i \omega)^{\alpha}+\mathbb{V}(i \omega)^{\beta}}
$$

By separating out the real and imaginary parts and substituting the definition for the charac- 
teristic time scale $\tau_{c}$ from Eq. A.13, we obtain

$$
G^{\prime}(\omega)=G_{0} \frac{\left(\omega \tau_{c}\right)^{\alpha} \cos (\pi \alpha / 2)+\left(\omega \tau_{c}\right)^{2 \alpha-\beta} \cos (\pi \beta / 2)}{\left(\omega \tau_{c}\right)^{2(\alpha-\beta)}+2\left(\omega \tau_{c}\right)^{\alpha-\beta} \cos (\pi(\alpha-\beta) / 2)+1}
$$

and

$$
G^{\prime \prime}(\omega)=G_{0} \frac{\left(\omega \tau_{c}\right)^{\alpha} \sin (\pi \alpha / 2)+\left(\omega \tau_{c}\right)^{2 \alpha-\beta} \sin (\pi \beta / 2)}{\left(\omega \tau_{c}\right)^{2(\alpha-\beta)}+2\left(\omega \tau_{c}\right)^{\alpha-\beta} \cos (\pi(\alpha-\beta) / 2)+1}
$$

where $G_{0} \equiv \mathbb{V} \tau_{c}^{-\alpha}$ sets the scale of the stress in the material. The model predictions for the storage and loss modulus from the FMM are plotted as dashed lines in Fig. 2(b). This shows that frequency domain predictions of this four-parameter model are still consistent with the data available. The second relaxation mode with $\mathbb{V}=4.7 \times 10^{6} \mathrm{~Pa} \mathrm{~s}^{\alpha}$ and $\alpha=0.65$ predicts a more rapid roll-off in the viscoelastic moduli at low frequencies $\left(\omega \ll \tau_{c}\right)$ or long times $\left(t \gg \tau_{c}\right)$, consistent with the evolution in the stress relaxation modulus $G(t)$ observed in Fig. 3(a).

In contrast to the Scott Blair model, the Fractional Maxwell Model can predict a crossover frequency $\omega_{c}$ at which $G^{\prime}(\omega)=G^{\prime \prime}(\omega)$, depending on the values of $\alpha$ and $\beta$. This crossover frequency is sometimes (incorrectly) referred to as a gelation criterion, but marks a transition from viscously-dominated regime $\left(\omega \leq \omega_{c}\right)$ to elastically-dominated $\left(\omega \geq \omega_{c}\right)$. This cross-over frequency can be calculated by equating Eq. (10) and (11) resulting in the following expression

$$
\omega_{c}=\left(\frac{\mathbb{G}}{\mathbb{V}}\left[\frac{\sin (\pi \alpha / 2)-\cos (\pi \alpha / 2)}{\cos (\pi \beta / 2)-\cos (\pi \beta / 2)}\right]\right)^{\frac{1}{\alpha-\beta}}=\frac{1}{\tau_{c}}\left[\frac{\sin (\pi \alpha / 2)-\cos (\pi \alpha / 2)}{\cos (\pi \beta / 2)-\cos (\pi \beta / 2)}\right]^{\frac{1}{\alpha-\beta}}
$$

and is real-valued provided $0 \leq \beta<0.5<\alpha \leq 1$ (as is always obtained for the cheese samples studied here). For the full-fat cheese shown in Fig. 2(b), substitution of the values for the quasi-properties and fractional exponents gives a value for the cross-over frequency of $\omega_{c}=1 \times 10^{-4} \mathrm{rad} \mathrm{s}^{-1}$. For cases where the system is predominantly elastic $(0 \leq \beta<\alpha<0.5)$ or viscous $(0.5 \leq \beta<\alpha \leq 1)$ over the entire frequency domain, no characteristic cross-over frequency exists.

To obtain analytical expressions for the creep compliance $J(t)$, the steps outlined above 
for $G(t)$ are repeated, but now with a step shear stress $\sigma(t)=\sigma_{0} H(t)$ as the input and solving for the resulting strain $\gamma(t)$. For the Scott Blair model this gives the following expression for the compliance (Jaishankar and McKinley, 2013):

$$
J(t) \equiv \frac{\gamma(t)}{\sigma_{0}}=\frac{1}{\mathbb{V}} \frac{t^{\alpha}}{\Gamma(1+\alpha)}
$$

and for the fractional Maxwell model

$$
J(t)=\left(\frac{1}{\mathbb{V}} \frac{t^{\alpha}}{\Gamma(1+\alpha)}+\frac{1}{\mathbb{G}} \frac{t^{\beta}}{\Gamma(1+\beta)}\right)
$$

In Fig. $3(\mathbf{b})$ we plot the measured and predicted creep compliance $J(t)$ for zero-fat and full-fat cheese at $10^{\circ} \mathrm{C}$. The SB model correctly predicts the measured creep compliance in both the zero-fat and full-fat samples from the material parameters obtained independently from the frequency sweep. Extending the SB model to a Fractional Maxwell Model is not required here because the data does not extend beyond $t_{\text {max }} \simeq 10^{2} \mathrm{~s}$.

From the data shown in Fig. 2 and 3 we have demonstrated that cheese, a fat-filled casein gel, displays power-law relaxation behaviour over a range of temperatures and compositions. The fractional constitutive framework allows us to predict the response of the cheese samples to standard shearing deformations. The wide range of microstructural scales in the material result in a very broad spectrum of relaxation timescales. These can compactly be represented in terms of a power-law relaxation spectrum or a Scott Blair element, and it is necessary to determine just two constitutive parameters to make the predictions. These material parameters are obtained by fitting the appropriate fractional expression for the standard linear viscoelastic material functions to the measured data. In Part I of this work we have additionally shown how this fractional constitutive framework can be used to derive equations for the texture attributes of firmness $(F)$, springiness $(S)$ and rubberiness $(R)$. These equations are uniquely expressed in terms of the quasi-property $\mathbb{G}$, and the fractional exponent $\beta$ of the Scott Blair element and for completeness summarized here in Table A.1. 


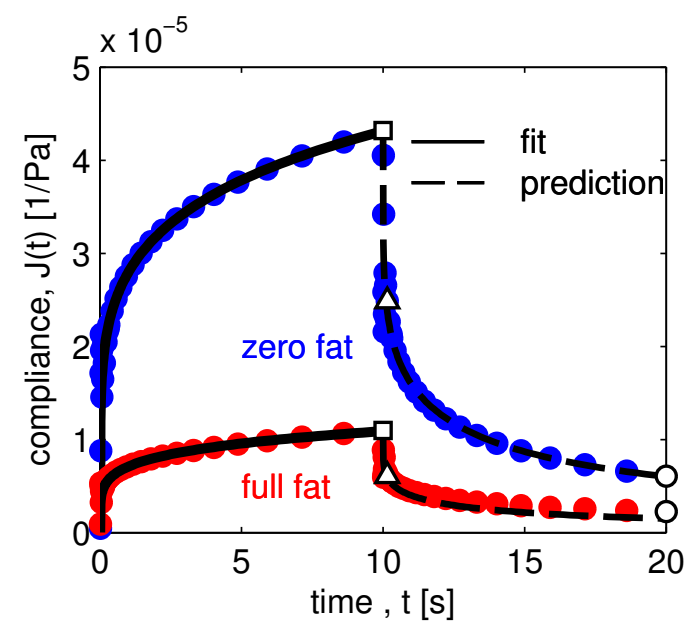

(a)

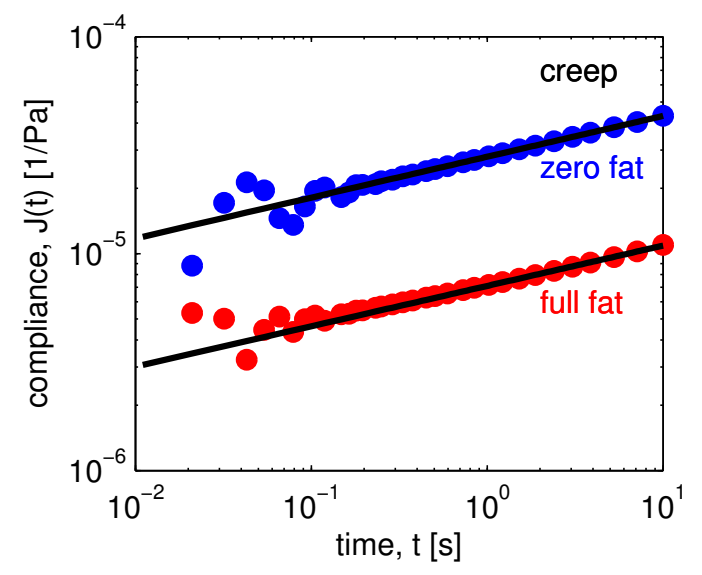

(c)

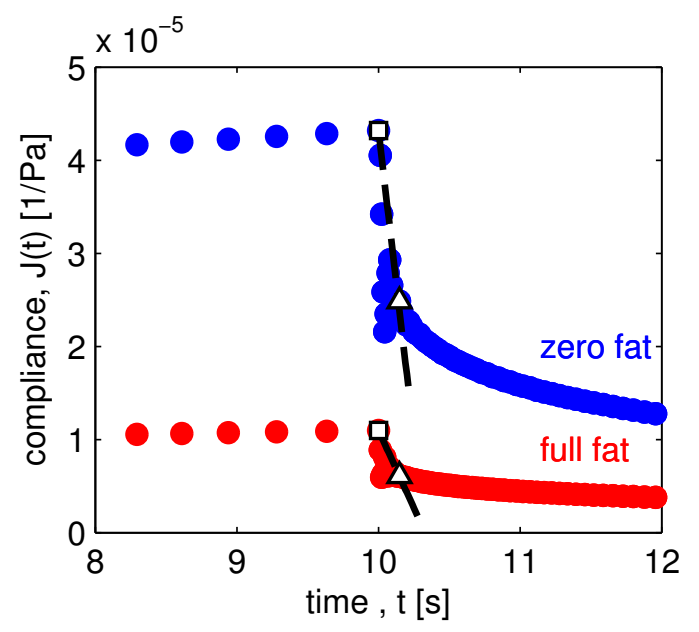

(b)

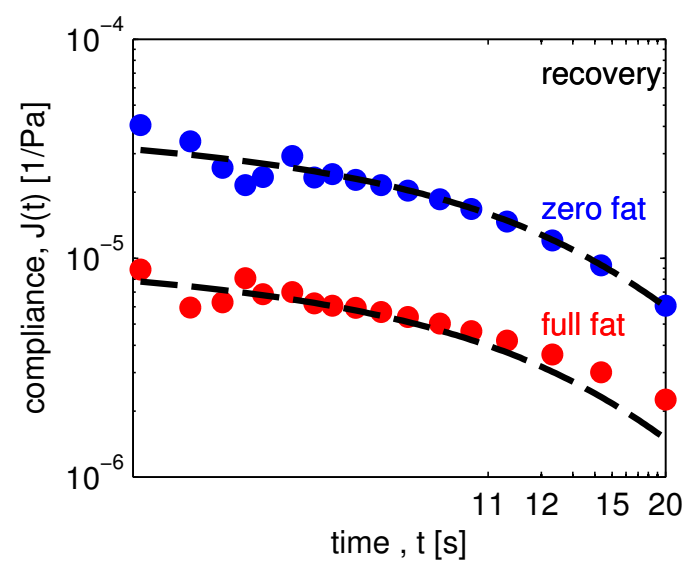

(d)

Figure 4: (a) Creep / recovery experiment $\left(\sigma_{0}=100 \mathrm{~Pa}\right)$ of zero-fat and full-fat cheese at $T=10^{\circ} \mathrm{C}$. Dashed line: prediction of the compliance $J(t)$ in the recovery phase using the Scott Blair element, Eq. (A.11), with $t_{f}=10 \mathrm{~s}$. The material parameters $\mathbb{G}$ and $\beta$ are obtained by fitting Eq. (13) to the compliance $J(t)$ of the creep phase. The fit result is indicated by the solid line and denoted as "SB fit". The hollow symbols are the specific points from the creep / recovery curve which are used to calculate the measured firmness $F$, (square), springiness $S$, (triangle), and rubberiness $R$, (circle). (b) Same experiment as in (a) now plotted over a limited time range $-2<\Delta t=t-t_{f}<2$, The absolute slope of the secant (dashed line) represents the springiness and is calculated with Eq. A.18). (c,d) Same experiment as in (a) plotted on a log-log scale and with the creep phase (c) and the recovery phase (d) separated. Both phases show some 'creep ringing' due to coupling of the elasticity and the moment of inertia of the instrument. (d) The compliance ultimately approaches zero. For full-fat cheese, the Scott Blair model overestimates the recovery, an irreversible flow event appears to have occurred, see section 3.3 These plots demonstrate that our equations correctly predict the firmness, springiness, and rubberiness of power-law materials such as cheese, from the two constitutive material parameters $\mathbb{G}$ and $\beta$ describing the material. 


\subsection{Fractional equations for firmness, springiness, and rubberiness (FSR)}

In section 3.1, we presented the fractional expression for the creep compliance $J(t)$ (Eq. (A.9) ) and showed that we can use this equation to obtain the material parameters $\mathbb{G}$ and $\beta$ for a given cheese. Our FSR-equations consist of only these material parameters and a specification of the time of measurement. This suggests that we can also determine values for both springiness and rubberiness by performing a measurement of the compliance $J(t)$; as long as we stay within the linear viscoelastic region; a separate recovery measurement is not required, although in principle it is easy to perform as a second step of an automated sequence immediately after a creep loading. Fig. 4 shows that this is indeed the case. The solid lines in Fig. $4(\mathbf{a})$ and (c) are fits of Eq. (13), the dashed lines in figures Fig. 4(a) and (d) are predictions from Eq. A.11). Fig. 4(a) shows that we correctly predict the rubberiness (circles) of the zero-fat and full-fat cheese, the latter being the most rubbery. In Fig. 4(b) we have plotted the same experiment as in $4(\mathbf{a})$ but now we zoom in on the region over which we measure springiness just after the time $t_{f}$. The secant lines plotted in Fig. 4(b) are predictions from Eq. A.18) and show that the zero-fat cheese is the springiest (with the largest rate of recovery), which can be ascribed to its smaller value of the quasi-property $\mathbb{G}$.

The equations for firmness, springiness, and rubberiness assume a viscoelastic response that can be described with a single springpot or Scott Blair element. These expressions contain only two material parameters, the quasi-property $\mathbb{G}$ and the fractional exponent $\beta$, and therefore we call these expressions (Eqs. A.15], A.18, , A.21]) the two-parameter FSRequations. In section 3.1 we have seen that for longer test durations, the addition of a second springpot in series (capturing a separate spectrum of relaxation processes) might be required for a more accurate fit or prediction. Following the same procedure as outlined above, but using the constitutive equation for the Fractional Maxwell Model (FMM) given in Eq. A.2], we can derive four-parameter formulations of the FSR-equations. The firmness is calculated by taking the inverse of the predicted compliance $J(t)$ of the FMM model Eq. (14), and substituting the fitted material parameters and chosen time of observation, $t_{f}$. To evaluate the rubberiness and springiness we need the expression for viscoelastic recoil of the Fractional 
Maxwell Model, which is given by (Jaishankar and McKinley, 2014):

$$
J(t)=\frac{t^{\beta}-\left(t-t_{f}\right)^{\beta}}{\mathbb{G} \Gamma(1+\beta)}+\frac{t^{\alpha}-\left(t-t_{f}\right)^{\alpha}}{\mathbb{V} \Gamma(1+\alpha)}, \text { for } t>t_{f}
$$

Substituting the appropriate times of observation and using the mathematical definitions for springiness Eq. (A.17), and rubberiness Eq. (A.20), gives numerical values for these attributes, Eq. A.23 and Eq. A.27 respectively. The response for recovery times $\Delta t>>t_{f}$ is dominated by the more viscous element and can be approximated by (Jaishankar and McKinley, 2014):

$$
J(t) \approx \frac{t_{f}^{\alpha}}{\mathbb{V} \Gamma(\alpha)}\left(\frac{t}{t_{f}}\right)^{\alpha-1}
$$

\subsection{Stress-time and flow}

We have argued that determining the creep / recovery curve, introduced to food rheology by Davis (1937), should be the standard rheological test protocol for defining and measuring the firmness, springiness, and rubberiness of food gels. The shape of the curve (and thus the magnitude of $F, S$ and $R$ ) is determined by the intrinsic material properties of the test material, as well as the severity of the creep loading. The latter is determined by setting both the magnitude $\left(\sigma_{0}\right)$ as well as the duration $\left(t_{f}\right)$ of the shear stress applied. Davis (1937) denoted the combination chosen as the 'stress-time', with units of Pa s. He showed that setting a high or low stress-time had significant effects on the amount of irreversible flow measured in the material, and thus on the measured food properties. In this section we demonstrate that the FSR-equations correctly predict the magnitude of $F, S$ and $R$, irrespective of the 'stress-time' conditions chosen.

First we look at zero-fat cheese and the linear viscoelastic responses. We have defined compliance-based expressions for the firmness, springiness, and rubberiness of food gels, thus in the linear viscoelastic regime $F, S$ and $R$ are independent of the stress applied. Davis (1937), however, based his conclusions on the flowing properties of cheese on stress-strain curves. To show how the magnitude of $F, S$ and $R$ vary if we were to take the same approach we also examine the effect of stress-time on the magnitude of strain-based definitions of the 
three texture attributes. We denote these alternate measures as $\hat{F}, \hat{S}$ and $\hat{R}$ respectively. Using such strain-based equations is equivalent to assessing $F, S$ and $R$ based on observations by vision only (Bourne (2002); Ewoldt (2013), see Fig. 1(a) in Faber et al. (2015)), without any (tactile) feedback of the stress applied.

The concept of stress-time has recently received a lot of attention in soft matter science, specifically in the study of yielding, where a progressive collapse of the internal structure gives rise to flow. The interplay of stress and time has been used to introduce concepts such as 'delayed yielding' (Sprakel et al., 2011) and 'time-stress superposition' (TSS) (Gobeaux et al. 2010). However, these two concepts have been applied in the polymer and plastics community for several decades (Matz et al., 1972) and predictive constitutive models have been successfully built on the basis of TSS (Engels et al., 2012).

To illustrate the concept and the consequences, we compare the firmness, springiness, and rubberiness of full-fat cheese at two stress-time conditions, one of which leads to yielding and irreversible flow. In the latter case our equations for $F, S$ and $R$ still correctly predict the measured value of firmness, springiness, and rubberiness. The three curves shown in Fig. 5 (a) are creep / recovery measurements on zero-fat cheese performed at three increasing levels of stress-time. The three cases are: 1) a firmness measuring time $t_{f}=10 \mathrm{~s}$ at a stress amplitude of $\sigma_{0}=100 \mathrm{~Pa}$; 2) a measurement time of 100 seconds combined with $100 \mathrm{~Pa}$; 3) 100 seconds at a stress level of $1000 \mathrm{~Pa}$. The times are chosen such that $t_{f 1}=t_{f 2} / 10=t_{f 3} / 10$, and the stresses such that $\sigma_{0,1}=\sigma_{0,2}=\sigma_{0,3} / 10$. Note that we have taken identical creep and recovery times within each experiment, thus the time for measuring rubberiness $\Delta t_{r}=t_{f}$. In Fig. $5(\mathbf{b})$ we have converted the raw strain $\gamma(t)$ measured for each case to the creep compliance $J(t)$, by dividing each case with the corresponding stress amplitudes. In Fig. 5(c) and (d) we separate the creep compliance from the recovery phase and plot both phases on a log-log scale, to reveal the presence of power-law creep. In Fig. 5 (e)-(h) the zero-fat cheese (blue symbols) is replaced by full-fat cheese (red symbols).

Curve 2 and curve 3 in Fig. $5(\mathbf{c})$ show identical compliances at the end of the curves. We thus measure the same firmness for stress-time conditions 2 and 3 and we have $F_{2}=F_{3}$ (any off-set in Fig. 5(b) comes from experimental error). This is what we would expect, even 
(a)

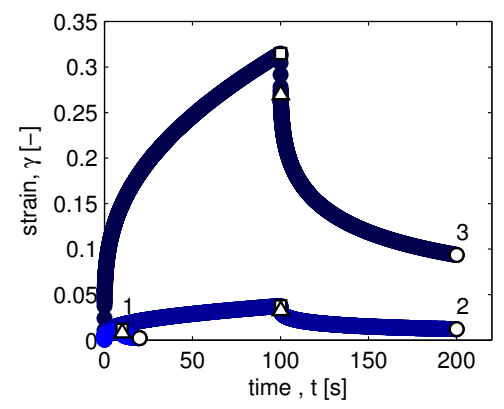

(b)

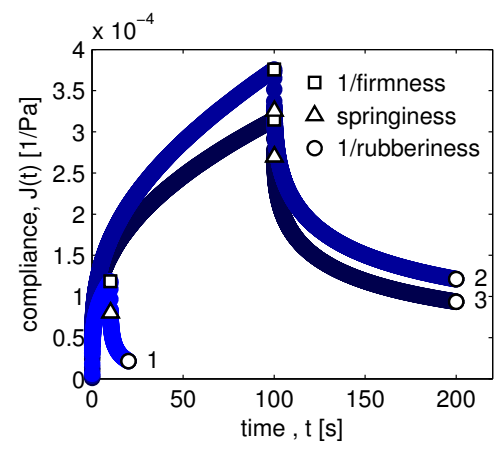

(c)

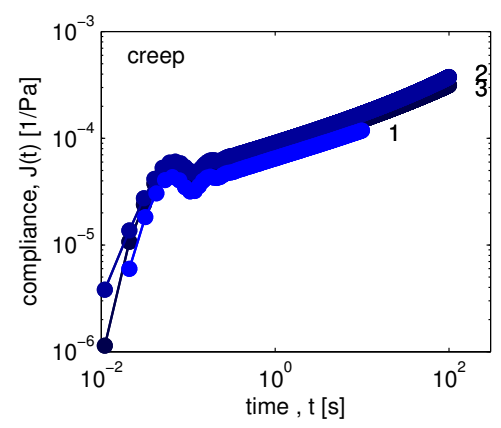

(d)

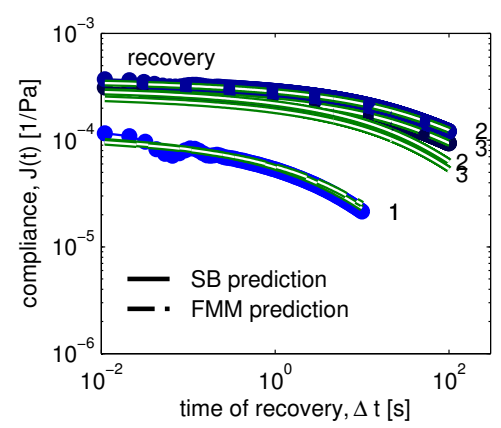

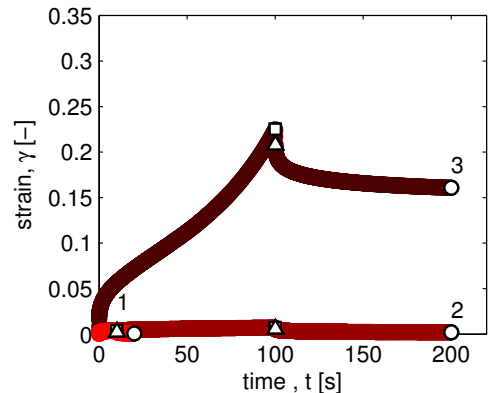

(e)

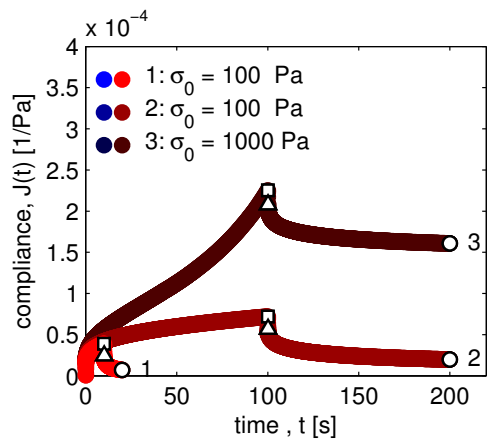

(f)

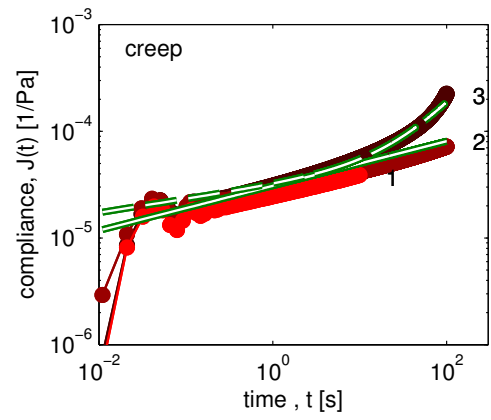

(g)

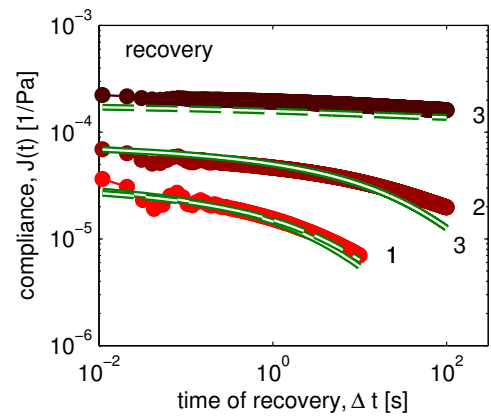

Figure 5: Creep / recovery experiments of zero-fat (blue) and full-fat cheese (red) at $25^{\circ} \mathrm{C}$ for three combinations of stress and time. The hollow symbols indicate the points on the creep / recovery curve that are used to calculate firmness (square), springiness (triangle) and rubberiness (circle). The numbers ' 1 ', ' 2 ' and ' 3 ' correspond to the order of severity of the stress-time loading. (a),(e) Strains $\gamma(t)$, (b),(f) converted to creep compliances $J(t)$. (c),(g) Same creep curves as in (b),(f) plotted on a log-log scale. (c),(g) Same recovery curves as in (b),(f) plotted on a log-log scale. (c) For zero-fat cheese all the curves coincide and the elastic recovery shown in (d) is well predicted with the Scott Blair model (single springpot). For full-fat cheese (g) enhanced flow sets in when a very high 'stress-time' is applied as shown in curve 3 . This leads to poor fits (g) and poor predictions (h) of the simple SB element (continuous lines), the Fractional Maxwell Model (dashed lines) gives good fits and better predictions however. 
though $\sigma_{0,2} \neq \sigma_{0,3}$, since in the linear viscoelastic region, firmness is independent of the stress, and both the test temperature and test time are identical $\left(t_{f 2}=t_{f 3}\right)$. Our definition of firmness, Eq. (A.15), and the fractional expression for the compliance, Eq. (13), allow us to calculate any point on the creep compliance curve with the expression:

$$
J(t)=\frac{1}{F_{2}}\left(\frac{t}{t_{f 2}}\right)^{\beta} \text { for } t<t_{f}
$$

When we substitute $t_{f 1}$ in Eq. (17) and take $F_{1}=1 / J\left(t_{f 1}\right)$, we can correlate the firmness for the three stress-time cases as:

$$
\left(\frac{t_{f 2}}{t_{f 1}}\right)^{\beta} F_{1}=F_{2}=F_{3}
$$

For $\beta=0.26$ and $t_{f 2} / t_{f 1}=10$, this gives a firmness $F_{1}$ that is 1.8 times smaller than $F_{2}$ and $F_{3}$, which corresponds to what is shown in Fig. 5(b). Such an effect of the stress-time loading on firmness is in line with Scott Blair's findings: only the time taken to load a material affects the judgement of firmness, variations in the stress applied have no effect (Scott Blair et al., 1947), at least within the material's linear regime. The magnitude of these temporal effects are determined by the scaling factor in Eq. (18), and the fractional exponent $\beta$. For a purely elastic material with $\beta=0$, this time effect is zero, for a purely viscous material, with $\beta=1$ the time effect is equal to $t_{f 2} / t_{f 1}$. If we defined firmness as the inverse of the strain at the end of creep and called it $\hat{F}$ than we would get:

$$
\left(\frac{t_{f 3}}{t_{f 1}}\right)^{\beta} \frac{\sigma_{0,3}}{\sigma_{0,1}} \hat{F}_{1}=\frac{\sigma_{0,3}}{\sigma_{0,1}} \hat{F}_{2}=\hat{F}_{3}
$$

For zero-fat cheese values in this experiment we have $18 \hat{F}_{1}=10 \hat{F}_{2}=\hat{F}_{3}$ which corresponds to the relative vertical position of the maximal strains $\gamma$ in Fig. 5(a).

Springiness can be graphically distinguished in the curves of the creep compliance, Fig. 5(b), as the distance between the values of the measured compliance at $t_{f}$, and $t_{f}+\Delta t_{s}$ (corresponding to the squares and the triangle respectively). Visually, it appears to be equal for all three stress-time conditions, so neither time nor stress has a significant effect on springiness. Numerical values of the springiness from the data in Fig. 5 (b), give $S_{1}=3.5 \times 10^{-4}$, 
$S_{2}=4.6 \times 10^{-4}, S_{3}=4.3 \times 10^{-4}(\mathrm{~Pa} \mathrm{~s})^{-1}$ so variations are less then $25 \%$.

This is what we predicted from Eq. A.18): at short times in the recovery phase $\left(\Delta t_{s}=0.1\right.$ s), springiness is only a function of the fractional exponent $\beta$ and the quasi-property $\mathbb{G}$. So for the three cases, the springiness can be calculated from the expression:

$$
S_{1}=S_{2}=S_{3}=\left|\frac{-10^{-1(\beta-1)}}{\mathrm{G} \Gamma(1+\beta)}\right|
$$

If we defined springiness from measured deformations instead of compliances, we would have obtained:

$$
\hat{S}_{1}=\hat{S}_{2}=\frac{\sigma_{0,3}}{\sigma_{0,1}} \hat{S}_{3}
$$

This is what we observe in Fig. $5(\mathbf{a})$, the vertical distance between the square and the triangle is 10 times larger for curve 3 than for curve 2 .

In part I of this work we demonstrated that the rubberiness $R$, Eq. (A.21), depends on two times, the creep time $t_{f}$ elapsed before measuring the firmness during creep and the subsequent elapsed time $\Delta t_{r}=t_{r}-t_{f}$ taken to measure rubberiness during recovery. In our stress-time experiments we have chosen $t_{f}$ to be equal to $\Delta t_{r}$ (this is true for all three curves for each cheese shown in Fig. 5. Substituting this in Eq. A.21) gives

$$
R_{1}=R_{2}=R_{3}=2-2^{\beta}
$$

We have defined rubberiness from a ratio of two compliances as $R=1-J\left(t_{f}+\Delta t_{r}\right) / J\left(t_{f}\right)$. Converting this into a definition based on a ratio of two strains, $\hat{R}=1-\gamma\left(t_{f}+\Delta t_{r}\right) / \gamma\left(t_{f}\right)$, requires both compliances to be multiplied by the same stress $\sigma_{0}$, which therefore cancels out. Thus $R=\hat{R}$.

For the firmness, springiness, and rubberiness of full-fat cheese we follow the same analysis as for the zero-fat cheese sample. The curves 1 and 2 in Fig. 5(e) and (f) show that for stress-time cases 1 and 2, equations (18)-(22) apply. However for stress-time case 3, with a long test time $t_{f}=100 \mathrm{~s}$ at a large stress of $\sigma_{0}=1000 \mathrm{~Pa}$, the creep response of curve 3 in Fig. 5(e)-(g) evidently deviates from a single power-law at long times. At such large stress- 
loadings, the two-parameter Scott Blair model for the creep compliance $J(t)$ is insufficient to describe the full range of retardation processes in the material, and the material begins to flow irreversibly at long times. To describe this flow we fit the data to the functional form predicted by the four-parameter Fractional Maxwell Model, given by Eq. (14) and Eq. A.10). The dashed line denoted with a ' 3 ' in Fig. 5(g) shows that this gives a greatly improved fit at large stress-times. This also leads to good prediction of the recovery data, as demonstrated by the dashed line labeled ' 3 ' in Fig. 5(h). We emphasize here again that this prediction is obtained by substituting a priori fitted material parameters for the FMM model into the corresponding expression for the recovery phase, Eq. (15). The more elastic element has a quasi-property value of $\mathrm{G}=0.4 \times 10^{5} \mathrm{~Pa} \mathrm{~s}^{\beta}$ and a fractional exponent $\beta=0.13$. The second element is a viscous dashpot: $\mathbb{V}=7.6 \times 10^{5} \mathrm{~Pa} \mathrm{~s}^{\alpha}$ and $\alpha=1$ (see Table A.2). One can say that the first element describes the semi-solid, fractional viscoelastic nature of cheese, whereas the second element (which dominates at long times) represents the ultimate transition of the fat-filled material at large values of the stress-time to a liquid with a very high viscosity.

The stress-induced yielding and failure of casein gels has recently been studied in detail by Leocmach et al. (2014), who combined creep experiments with ultrasonic velocimetry imaging. They showed that at large strains, casein gels display brittle-like failure which is a resultant of two consecutive physical processes. The first is reversible primary creep, also referred to as Andrade creep (Andrade, 1910). In contrast to crystalline or amorphous solids, the strain in viscoelastic gels can be fully recovered during primary creep (Leocmach et al., 2014), which agrees with the prediction of the SB element. The second process is nucleation and growth of fractures, leading to plastic deformation and eventually complete sample failure. This regime is commonly called tertiary creep. In between lies a transition regime which is denoted as secondary creep.

If we map these processes onto our measurements and model predictions, we can conclude that the more elastic SB element in the FMM model (with parameters $\mathbb{G}$ and $\beta$ ) represents, in compact form, the spectrum of reversible viscoelastic processes in the material, and the second element in series (with parameters $\mathbb{V}$ and $\alpha$ ) models empirically any additional ir- 
reversible plastic part. By arranging the elements in series we additively combine the elastic and plastic strains that result from each physical process. When fitting the FMM to rheometric creep data obtained at large stress-times (which may contain a yielding event) we are using a model derived from linear viscoelastic theory to capture a non-linear viscoelastoplastic response. It is important to recognize that a linear model such as the Fractional Maxwell Model does not predict a yield stress or strain, this requires more sophisticated non-linear models that include a damping function (Jaishankar and McKinley, 2014) or a damage function (Tanner et al., 2008; Mohammed et al., 2013). However, fitting creep data at small and moderate strains to the FMM enables us to effectively predict the recovery phase of the bulk material very well (compare the five curves in Fig. $5(\mathbf{d})$ and (h)), suggesting that it also correctly describes two of the primary processes occurring during the initial loading and unloading phases. We will use this feature to our advantage to separate viscoelastic, recoverable contributions to the firmness, springiness, and rubberiness, from the additional plastic, non-recoverable contributions that occur in the case of irreversible flow in the material.

We have demonstrated that our definitions for the firmness $F$, springiness $S$, and rubberiness $R$, of food gels give correct quantitative predictions of the effect of the stress-time loading on measurements of $F, S$, and $R$. If the deformation amplitude remains within the linear viscoelastic regime, then the magnitude of the springiness is not affected by stress or time. For our chosen times of observations for the firmness and rubberiness such that $t_{f}=\Delta t_{r}$, the texture attribute of rubberiness $R$, becomes stress- and time-independent as well. When very large stress-time loading leads to larger deformations in the viscoelastoplastic region, i.e. resulting in strains in the material that are larger then the yield strain, our four-parameter FSRequations derived from the Fractional Maxwell Model (FMM) correctly predict the variation in the firmness, springiness, and rubberiness of cheese, provided that the material has not entered the regime of tertiary creep and fracture. The presence or absence of an irreversible flow event at large values of the stress-time, has a large impact on quantitative rheological measurement of the firmness, springiness, and rubberiness of food gels. This suggests that when the firmness, springiness, and rubberiness of a food gel is evaluated by hand, the magnitude of the stress-time loading applied should be defined as precisely as possible, conforming to 
Scott Blair's initial assertion (Scott Blair and Coppen, 1940b). 


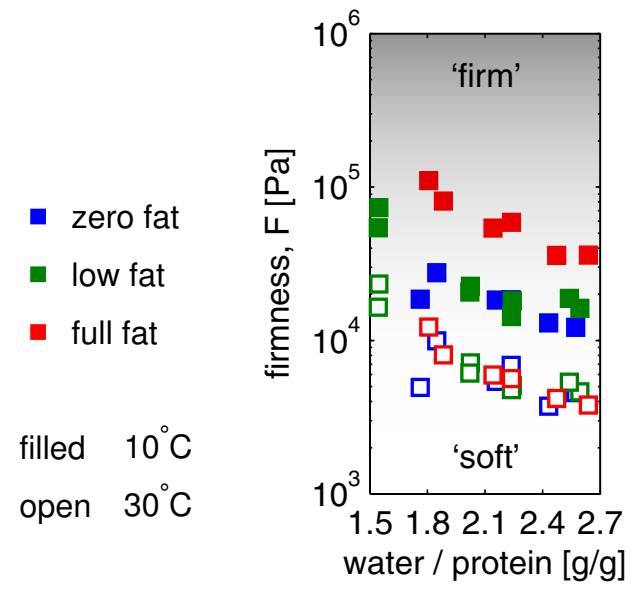

(a) Firmness

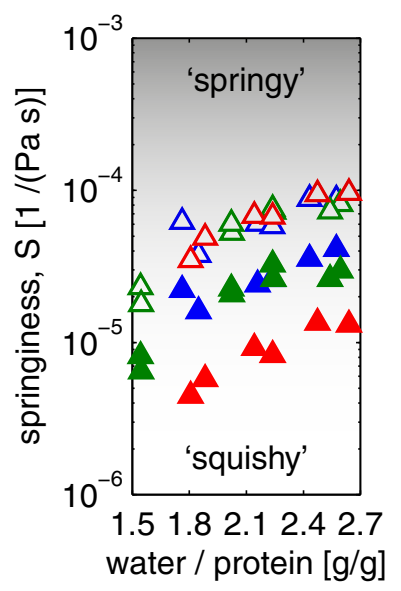

(b) Springiness

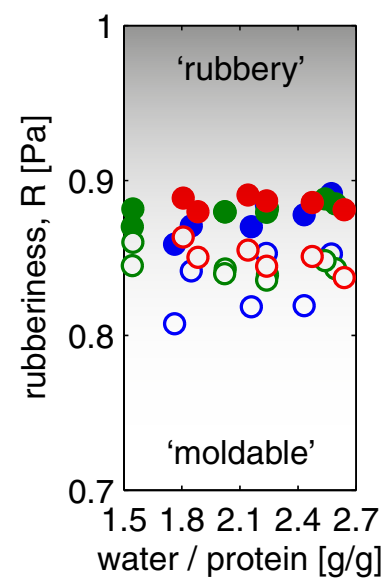

(c) Rubberiness.

Figure 6: Composition-temperature-texture plots of Gouda cheese. (a) firmness $F$, (b) springiness $S$, (c) rubberiness $R$. The plots are obtained by determining the quasi-property $\mathbb{G}$, and the fractional exponent $\beta$, from forty frequency sweeps, and entering the value in the FSR-equations. The time of observations for firmness and rubberiness are $t_{f}=\Delta t_{r}=10 \mathrm{~s}$. For springiness $\Delta t_{s}=0.1 \mathrm{~s}$. (a) Cheese is most firm at low temperature, and at a low water / protein ratio, and high fat content. (b) Springiness is inversely related to firmness, the softest cheese is the most springy. (c) Rubberiness is a function of the fractional exponent only.

\subsection{Comparing cheese formulations on the basis of firmness, springiness, and rubberiness}

In Fig. 2 we showed four representative frequency sweeps of Gouda cheese, varying in temperature and composition. In all cases the cheese showed a clear power-law relaxation. This relaxation behaviour can be adequately described by the Scott Blair model for the complex modulus, Eq. (2). By fitting this model to the dataset, we determine the numerical values of the two material properties that characterize this cheese: the quasi-property $\mathbb{G}$ and the fractional exponent $\beta$. From these material parameters we can calculate firmness $F$, springiness $S$, and rubberiness $R$, at any time of observation, using equations (A.15), A.18) and A.21.

We demonstrate the power of these FSR-equations in Fig. 6. In total we have measured 40 combinations of cheese composition and temperature, corresponding to 80 curves of the frequency-dependent storage and loss modulus, $G^{\prime}(\omega)$ and $G^{\prime \prime}(\omega)$. We condense this extensive dataset into the three composition-temperature-texture plots of the firmness, springiness, and rubberiness, shown in Fig. 6. The definitions for FSR, in equations A.15, A.18) and (A.21) allow us to enter any time of observation of interest; here we select $t_{f}=\Delta t_{r}=10 \mathrm{~s}$ for firmness and rubberiness, and $\Delta t_{s}=0.1 \mathrm{~s}\left(\ll t_{f}\right)$ for springiness determination. 
Compositional changes are represented in two ways. On the abscissa we have plotted the water/protein ratio $(w / p)$ of the cheese. Variations in fat content are represented by the three colors, blue, green and red, for zero, low and full-fat respectively. Since the material properties of many food products are highly temperature sensitive (Szczesniak, 1975), we have measured the viscoelastic properties of the cheese at $T=10^{\circ} \mathrm{C}$ (filled markers), a temperature relevant for storing cheese, and also at $T=30^{\circ} \mathrm{C}$ (unfilled markers), a temperature the cheese will attain during oral processing.

We interpret the data in this figure as describing the reformulation window for firmness. Under storage conditions the cheese has to be firm; this inhibits sagging and gives optimal slicing properties. Our reference point for optimal firmness prior to consumption is fullfat cheese at $T=10^{\circ} \mathrm{C}$, having its standard water/protein ratio of $w / p=1.8$. This appears to be the most firm cheese. Now the challenge for the food engineer dealing with product reformulation becomes apparent: at $T=10^{\circ} \mathrm{C}$ either a reduction of fat content, or an increase of water content, will always lead to a softer cheese. This will inevitably lead to inferior processing properties. When removing fat from the product for reasons of calorie reduction, one has to lower the water/protein ratio as a countermeasure to retain firmness.

When increasing temperature we see that our reference full-fat cheese shows a pronounced drop in firmness. It can be argued that this 'thermal-induced softening' gives rise to a melting sensation during consumption (Devezeaux de Lavergne et al., 2015). This further augments the 'stress-induced softening' we observed in Fig. 5(g). Removing fat significantly reduces this thermal softening-effect on cheese firmness. Changing the water/protein ratio has no effect on the temperature-firmness relation.

The strong 'thermal-induced softening' of full-fat, semi-hard cheese can be physically explained using filled gel models to describe the structure shown in Fig. 1(d). Yang et al. (2011) fitted a variety of these models to data for the storage modulus $G^{\prime}(\omega)$ of Cheddar cheese, at a frequency $\omega=1 \mathrm{rad} \mathrm{s}^{-1}$. Depending on the model applied, they predicted that at $T=10{ }^{\circ} \mathrm{C}$, the shear modulus, $G_{f}$, of the filler (fat) is 6-11 times larger than the shear modulus of the matrix $G_{m}$. At $T=10^{\circ} \mathrm{C}$ the filler thus acts as a firmness enhancer. When increasing the temperature of the cheese from $T=10^{\circ} \mathrm{C}$ to $T=25^{\circ} \mathrm{C}$ they predicted a 
decrease of the filler modulus $G_{f}$ by a factor ranging from 10-27, depending on the model applied. Over the same temperature range, $G_{m}$ decreased not more than a factor of two, which corresponds to what we measure for the decrease of the storage modulus of zero fat cheese when raising the measuring temperature from $T=10^{\circ} \mathrm{C}$ to $T=30{ }^{\circ} \mathrm{C}$ (Fig. 2) (a) and (c), blue filled symbols). At $T=25^{\circ} \mathrm{C}$, Yang et al. (2011) measure a storage modulus, $G^{\prime}(\omega)$, that is independent of the fat content of the cheese, which is similar to what we find for the firmness $F$, at $T=30{ }^{\circ} \mathrm{C}$ and a time of observation $t_{f}=10 \mathrm{~s}$. (Fig. 6(a), hollow symbols). Note that the firmness of low-fat cheese (green symbols in Fig. 6(a)), is approximately equal to the firmness of zero-fat cheese (blue symbols) at both temperatures. Apparently there is a threshold value for the fat volume fraction, $\Phi_{f}$, below which this cheese constituent has no significant effect on the overall firmness of the cheese.

Next we examine the effect of temperature and composition of the cheese on the springiness $S$, plotted in Fig. 6(b). We see that the softer cheese will be the most springy, which corresponds to the anti-correlation between firmness and springiness found in panel tests (Goh et al., 2003). The trend can be explained by the inverse relation between springiness and firmness, as expressed in Eq. (A.18), and has also been observed in the Texture Profile Analysis of reformulated muffins (Matos et al., 2014).

The rubberiness plot, Fig. $6(\mathbf{c})$, shows that $S$ and $R$ indeed are two distinct properties, and they scale differently with composition and temperature. Since we have chosen the time of observation $\Delta t_{r}$ for rubberiness to be equal to $t_{f}$, we can use Eq. (22) to calculate $R$. This expression shows that under these time conditions, the rubberiness $R$ is only a function of the fractional exponent $\beta$. For our cheeses, the measured values for this exponent range from $0.14 \leq \beta \leq 0.25$. Fig. 6 (c) shows that for these values the strain recovery is fast; in 10 seconds $80-90 \%$ of the strain is recovered for all samples.

We can conclude that reformulating a cheese by merely changing the relative amount of protein, water and fat will give great firmness deficits, both from a sensory texture as well as a processing perspective. To decouple this texture-composition relation, alternative structuring routes need to be explored. This exploration should start with a physical understanding of the observed scaling between fat content and water/protein ratio on the one hand, and firmness, 
rubberiness and springiness on the other. Such understanding requires structure-texture relations such as the filled gel models tested by Yang et al.(2011) or such as the model proposed by Bot et al. (2014), who developed a structural model for the firmness and syneresis of food gels as a function of composition and temperature based on a set of recursive power-law scaling relations. However both Yang et al. (2011) and Bot et al. (2014) do not account for the time-dependence of the magnitude of the firmness F, and the structure-texture relations they use have not been extrapolated to other texture attributes assessed at first touch and first bite. Since we have shown that the attributes $F, S$ and $R$, all stem from the quasi-property, $\mathbb{G}$, and the fractional exponent $\beta$ of the viscoelastic test material, a coupling of the models discussed by Bot et al. (2014) and Yang et al. (2011) to the magnitudes of the quasi-property $\mathbb{G}$, and fractional exponent $\beta$ of Scott Blair's springpot, would be the next step to take in the development of an appropriate structure-texture model.

\subsection{Practical relevance of firmness measurements}

In section 3.4 we demonstrated the use of the FSR-equations as a screening tool for texture optimisation. In Fig. 6 a wide range of formulations are plotted in texture-compositiontemperature space. The three plots show at a glance the reformulation window for cheese with respect to the firmness, springiness, and rubberiness. However the construction of these plots requires a substantial experimental effort, while a structure-texture model should reduce these efforts and point a priori towards the most optimal formulation and structure.

We have chosen to measure firmness, springiness, and rubberinessusing a stress-controlled experimental protocol. Such an experiment exposes temporal effects in the material more directly than the more common rate-controlled compression experiment used in Texture Profile Analysis (Friedman et al., 1963). Moreover, it enables us to interconnect the firmness $F$, to situations where stresses are applied for short times, such as sensory texture measurement, or for long times, such as in storing cheese (Fig. 1(a) in Part I, Faber et al. (2015)). We have developed rheological definitions of firmness, springiness, and rubberiness based on an understanding of compliances, since in the linear viscoelastic regime, the judgement of firmness is independent of the stress applied (Scott Blair and Coppen, 1942a). To extrapolate 


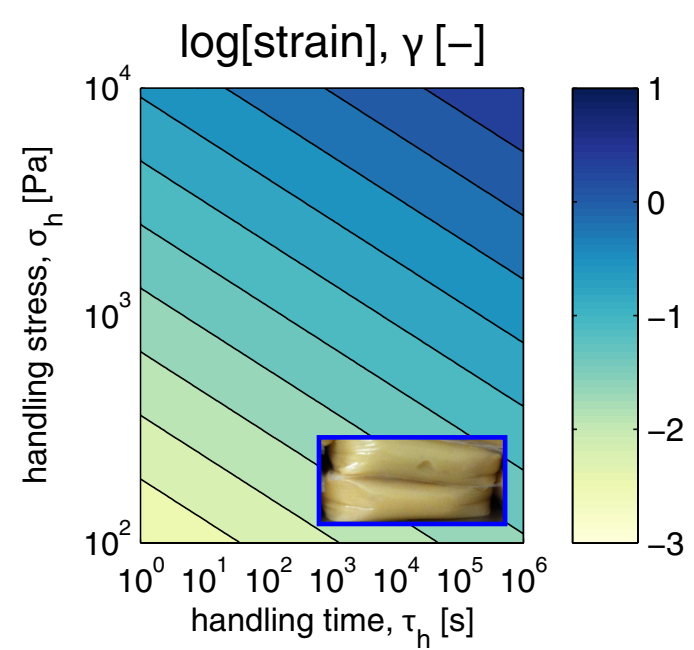

(a) zero fat

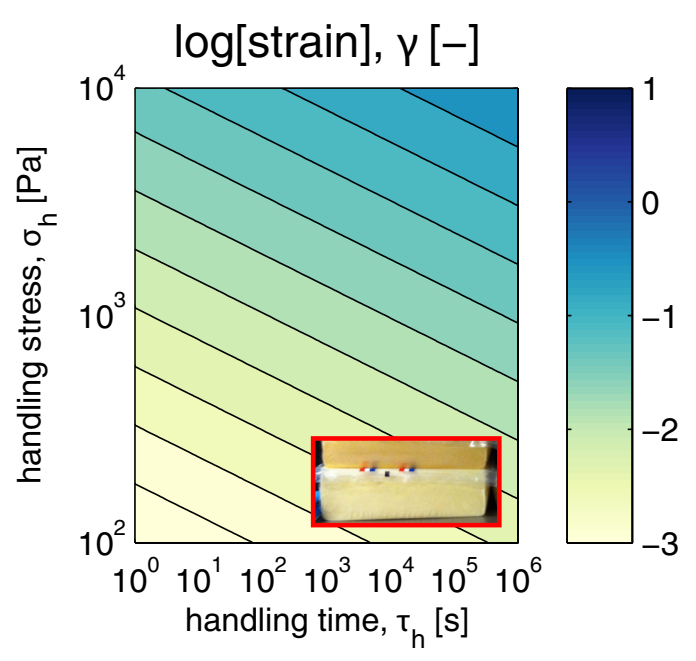

(b) full fat

Figure 7: The effect of handling stress, $\sigma_{h}$ and handling time $\tau_{h}$ on the predicted deformation of (a) zero fat and (b) full-fat cheese at $10{ }^{\circ} \mathrm{C}$. Shear strains, $\gamma$ are plotted as contours on a log-scale and calculated from Eq. 23. At equal stress-time loadings, the softer zero-fat cheese, $\left(F=0.2 \times 10^{5}\right)$ Pa generates lighter contours compared to the firmer full fat cheese, $F=1 \times 10^{5} \mathrm{~Pa}$, as a result of the higher strains accumulated. The slopes of the contours are equal to the fractional exponent $\beta$. For zero-fat cheese, $\beta=0.18$ and for full-fat cheese $\beta=0.14$. At stress-time loadings that lead to irreversible flow, Eq. 23 is not accurate and the predicted strains are under-estimated. Stress-time conditions for texture judgment are located in the top left corner of the plots, where irreversible flow is desired to prevent rubberiness. Stress-time conditions for storing cheese on the shelf are located on the bottom right corner of the plots, where higher levels of firmness favour shape retention.

the measured firmness to how a cheese performs under practical conditions, e.g. whether it will retain its shape when stored on a shelf, we need to convert a firmness measurement to an expected deformation. In section 3.3 we revisited the concept of stress-time, originally introduced by Davis (1937), and we showed how to calculate the resulting material strains $\gamma$ from the firmness $F$, for a certain stress-time loading profile. In Fig. 7 we plot the evolution in the sample strain as contours in stress-time space for creep loading of both zero-fat (a) and full-fat (b) cheese at $10^{\circ} \mathrm{C}$. The stress-time space is spanned by the range of typical handling stresses $\sigma_{h}$ and handling times $\tau_{h}$ common in the cheese industry. The contour lines for specific values of the strain $\gamma\left(\tau_{h}, \sigma_{h}\right)$ are calculated from the expression

$$
\gamma=\frac{\sigma_{h}}{F}\left(\frac{\tau_{h}}{t_{f}}\right)^{\beta}
$$

One can obtain insight into the practical relevance and implications of a firmness mea- 
surement by considering three terms in this expression. First, we focus on the product $\sigma_{h} \tau_{h}^{\beta}$ in the numerator, which is the measure of stress-time inherent to the handling process to which the cheese is subjected. When firmness is judged by hand, stresses are high and times are short, which corresponds to the upper left corner of each plot in Fig. 7. When a cheese is stored on the shelf, the imposed stresses (arising solely from body forces) are relatively low, but times can be of the order of weeks. This is represented by the lower right corner of the graph. Under these conditions the full-fat cheese depicted in Fig. 7(b) will be the least deformed, since it is the most firm.

The second term of interest in Eq. (23) is the ratio $\tau_{h} / t_{f}$ which is a dimensionless ratio of times in the spirit of Reiner's original definition of the Deborah number (Reiner, 1964). For cheese subjected to creep loading, the characteristic times in this ratio are the values inherent to the handling process to which the material is subjected and that over which the firmness is determined. For the cheese industry, handling times vary from $\tau_{h}=1 \mathrm{~s}$ (for firmness judged by hand) to $\tau_{h}=10^{6}-10^{7} \mathrm{~s}$ (for storing cheese on a shelf). For a time of observation $t_{f}=10$ $\mathrm{s}$ this gives a range for $\tau_{h} / t_{f}=10^{-1}-10^{6}$. A high value for $\tau_{h} / t_{f}$ implies that in the laboratory the rheologist is probing timescales much smaller than the practical time scale of relevance for the material and one should be careful of extrapolating conclusions from a linear viscoelastic measurement performed over a limited frequency range or a single firmness measurement.

Finally, we learn from Eq. 23) that the deformation of a cheese subjected to creep loading can be factorised in two parts. The first is an instantaneous deformation, observed after a very brief period of stress-time loading. The scale of the instantaneous deformation is set by $\sigma_{h} / F$. It is worth noting that the instrumental resolution of this instantaneous deformation is restricted by the response time of the rheometer used. The final deformation caused by the stress-time loading is obtained by multiplying $\sigma_{h} / F$ with the factor $\left(\tau_{h} / t_{f}\right)^{\beta}$. For a purely elastic material, $\beta=0$ and this factor is thus $\left(\tau_{h} / t_{f}\right)^{0}=1$. For such a material, viscoelastic effects are absent and all contour lines in Fig. 7 are horizontal. For a purely viscous material $\beta=1$ the effect of the elapsed time on strain is linear such that the iso-strain lines have a slope of -1 on this logarithmic plot.

The plots in Fig. 7 and Fig. 2 are generated using expressions that are derived from the 
canonical fractional constitutive equation of a single springpot or Scott Blair element (SB). In section 3.1 and 3.3 we have shown that for cases where the stress-time loading leads to a secondary relaxation process in the cheese, we are still able to predict the firmness, springiness, and rubberiness within the fractional constitutive framework. All we require is a generalization of the constitutive model with a second element in series, which yields the equation of the Fractional Maxwell Model, Eq. (A.2). In section 3.3 we have shown that fullfat cheese begins to flow plastically when subjected to high stress-time loadings. This failure may be proceeded by a secondary relaxation processes caused by the propagation of micro fractures in the material (Leocmach et al., 2014). Such microscopic phenomena of course cannot be captured by a bulk phenomenological model such as the FMM, but are captured empirically by the second viscous-like springpot element (with $\alpha \approx 1$ ). 


\section{Conclusion}

The FSR-equations we have developed for a typical power-law food gel contain only two material parameters, the quasi-property $\mathbb{G}$ (or scale factor) of the gel and the fractional exponent $\beta$ (Scott Blair, 1947). The resulting stress or strain in the viscoelastic material depends on the experimental time of observation $t_{o}$ and the loading protocol. The two material parameters that characterize the Scott Blair element can be extracted from measurements of any of the standard linear viscoelastic material functions: complex modulus, $G^{*}(\omega)$, storage modulus, $G^{\prime}(\omega)$, loss modulus, $G^{\prime \prime}(\omega)$, relaxation modulus, $G(t)$, or creep compliance, $J(t)$.

When a food gel is subjected to creep loadings, the final deformation of the gel depends on its intrinsic firmness and the severity of the loading. Davis (1937) introduced the concept of 'stress-time' to qualitatively discuss the effects of loading severity on cheese deformation. We have demonstrated that our equations for the firmness $F$, springiness $S$, and rubberiness $R$, of food gels give correct quantitative predictions of the effect of the stress-time loading on cheese deformation and the resulting values for $F, S$ and $R$. If the stress-time loading to which the cheese is subjected will cause the cheese to reach its yield strain during the creep phase, the material will start to behave in a viscoelastoplastic nature and our twoparameter FSR-equations (of Scott Blair type) cannot predict the recovery phase correctly. Four-parameter FSR-equations derived from the Fractional Maxwell Model appear to give much better descriptions of the post-yield, secondary creep response, by incorporating an irreversible viscous term (with viscosity $\eta=\mathbb{V}$ and $\alpha \approx 1$ ). This approach allows us to correctly predict the incomplete recovery of the material in the case of irreversible (plastic) flow contributions.

We have presented two methods of using the FSR-equations for product reformulation. The first is to measure the linear viscoelastic response for a wide range of product formulations, extract the material parameters $\mathbb{G}$ and $\beta$ of each formulation, and construct three composition texture plots. This reveals the operating window for reformulation when required levels for each texture attribute are available. Our analyses of different cheeses show that fat acts as the perfect filler in modulating firmness, springiness, and rubberiness for opti- 
mal texture and processability. At storage temperatures, filling a cheese with fat raises $\mathbb{G}$ and reduces the exponent $\beta$ compared to zero-fat cheese (see Figs 2 and 3). Both changes lead to an increase of firmness, springiness, and rubberiness (Fig. 6), ideal for maintaining shape. At eating temperature the melting of fat induces thermal softening, which will make the oral processing a more pleasant experience.

The second way of exploiting the FSR-framework is to create contour plots for either firmness, springiness or rubberiness, as one varies the two material parameters $\mathbb{G}$ and $\beta$ (see Part I, Fig.6). These plots direct the developer towards the desired combination of material properties instead of optimal composition. This is still one step removed from structure-texture engineering for product reformulation: i.e. a guided modification in the cheese microstructure in order to maintain texture while changing composition. What remains to be done is to connect the morphology of the cheese (see images in $1(\mathbf{c}),(\mathbf{d}))$ to the resulting macroscopic fractional constitutive response via an appropriate multiscale micromechanical model. We hope to report on suitable approaches to this texture engineering challenge in the near future. 


\section{References}

Abramowitz, M. and Stegun, A. (1964). Handbook of Mathematical Functions. U.S. National Bureau of Standards, Washington.

Andrade, E. N. D. C. (1910). On the viscous flow in metals and allied phenomena. Proceedings of the Royal Society of London. Series A, 84(567):1-12.

Bagley, R. L. and Torvik, P. J. (1983). Theoretical basis for the application of fractional calculus to viscoelasticity. Journal of Rheology, 27(3):201-210.

Bot, A., Foster, T. J., and Lundin, L. (2014). Modelling acidified emulsion gels as Matryoshka composites: Firmness and syneresis. Food Hydrocolloids, 34:88-97.

Bourne, M. C. (2002). Food Texture and Viscosity: Concept and Measurement, pages 1 -32. Food Science and Technology. Academic Press, London, second edition.

Bremer, L. G. B., Bijsterbosch, B. H., Schrijvers, R., van Vliet, T., and Walstra, P. (1990). On the fractal nature of the structure of acid casein gels. Colloids and Surfaces, 51(C):159170.

Davis, J. (1965). Cheese, volume I - Basic Technology. J. \&. A. Churchill Ltd., 1 edition.

Davis, J. G. (1937). 162. The rheology of cheese, butter and other milk products. (the measurement of "body" and "texture"). Journal of Dairy Research, 8:245-264.

De Kruif, C., Huppertz, T., Urban, V., and Petukhov, A. (2012). Casein micelles and their internal structure. Advances in Colloid and Interface Science, 171-172:36-52.

De Kruif, C. G. and Holt, C. (2003). Casein micelle structure, functions and interactions.

In Fox, P. F. and McSweeney, P. L. H., editors, Advanced Dairy Chemistry: 1. Proteins, volume 1, pages 233-276. Springer US, 3 edition.

Devezeaux de Lavergne, M., van Delft, M., van de Velde, F., van Boekel, M. A. J. S., and Stieger, M. (2015). Dynamic texture perception and oral processing of semi-solid food gels: Part 1: Comparison between QDA, progressive profiling and TDS. Food Hydrocolloids, 43:207-217.

Dickinson, E. (2012). Emulsion gels: The structuring of soft solids with protein-stabilized oil droplets. Food Hydrocolloids, 28(1):224-241. 
Engels, T. A. P., Govaert, L. E., and Meijer, H. E. H. (2012). Mechanical characterization of glassy polymers: Quantitative prediction of their short- and long-term responses. In Matyjaszewski, K. and Möller, M., editors, Polymer Science: A Comprehensive Reference, volume 2, pages 723-747. Elsevier.

Ewoldt, R. H. (2013). Extremely soft: Design with rheologically complex fluids. Soft Robotics, 1(1):12-20.

Faber, T. J., Jaishankar, A., and McKinley, G. H. (2015). Describing the firmness, springiness and rubberiness of food gels using fractional calculus. Part I: Theoretical framework. In preparation for Food Hydrocolloids.

Foegeding, E. A. and Drake, M. A. (2007). Invited review: Sensory and mechanical properties of cheese texture. Journal of Dairy Science, 90(4):1611-1624.

Foegeding, E. A., Vardhanabhuti, B., and Yang, X. (2011). Dairy systems. In Norton, I., Pyropoulos, F., and Cox, P., editors, Practical Food Rheology: An Interpretive Approach, pages 133-172. Wiley-Blackwell.

Friedman, H. H., Whitney, J. E., and Szczesniak, A. S. (1963). The texturometer - a new instrument for objective texture measurement. Journal of Food Science, 28(4):390-396.

Friedrich, C., Schiessel, H., and Blumen, A. (1999). Constitutive behavior modeling and fractional derivatives. In Siginer, D. A., Kee De, D., and Chhabra, R. P., editors, Advances in the Flow and Rheology of NonNewtonian Fluids, volume 8 of Rheology Series, pages 429-466. Elsevier.

Gobeaux, F., Belamie, E., Mosser, G., Davidson, P., and Asnacios, S. (2010). Power law rheology and strain-induced yielding in acidic solutions of type I-collagen. Soft Matter, 6(16):3769-3777.

Goh, S. M., Charalambides, M. N., and Williams, J. G. (2003). Mechanical properties and sensory texture assessment of cheeses. Journal of Texture Studies, 34(2):181-201.

Gunasekaran, S. and Ak, M. M. (2003). Cheese Rheology and Texture. CRC Press.

Jaishankar, A. and McKinley, G. H. (2013). Power-law rheology in the bulk and at the interface: Quasi-properties and fractional constitutive equations. Proceedings of the Royal Society A: Mathematical, Physical and Engineering Sciences, 469(2149). 
Jaishankar, A. and McKinley, G. H. (2014). A fractional K-BKZ constitutive formulation for describing the nonlinear rheology of multiscale complex fluids. Journal of Rheology, 58(6):1751-1788.

Johnson, M. and Law, B. A. (2010). The origins, development and basic operations of cheesemaking technology. In Law, B. and Tamime, A., editors, Technology of Cheesemaking, pages 68-97. Wiley-Blackwell.

Kloek, W. (1998). Mechanical properties of fats in relation to their crystallization. $\mathrm{PhD}$ thesis, Kloek, Wageningen.

Leocmach, M., Perge, C., Divoux, T., and Manneville, S. (2014). Creep and fracture of a protein gel under stress. Physical Review Letters, 113:038303.

Lucey, J. A., Mishra, R., Hassan, A., and Johnson, M. E. (2005). Rheological and calcium equilibrium changes during the ripening of cheddar cheese. International Dairy Journal, 15(6-9):645-653.

Luyten, H. (1988). The rheological and fracture properties of Gouda cheese. PhD thesis, Dairy Science, Wageningen University.

Matos, M. E., Sanz, T., and Rosell, C. M. (2014). Establishing the function of proteins on the rheological and quality properties of rice based gluten free muffins. Food Hydrocolloids, $35: 150-158$.

Matz, D. J., Guldemond, W. G., and Cooper, S. L. (1972). Delayed yielding in glassy polymers. Journal of Polymer Science: Polymer Physics Edition, 10(10):1917-1930.

McMahon, D. J., Paulson, B., and Oberg, C. J. (2005). Influence of calcium, pH, and moisture on protein matrix structure and functionality in direct-acidified nonfat mozzarella cheese. Journal of Dairy Science, 88(11):3754-3763.

Mohammed, M. A. P., Tarleton, E., Charalambides, M. N., and Williams, J. G. (2013). Mechanical characterization and micromechanical modeling of bread dough. Journal of Rheology, 57(1):249-272.

Ng, T. S.-K. and McKinley, G. H. (2008). Power law gels at finite strains: The nonlinear rheology of gluten gels. Journal of Rheology, 52(2):417-449.

O’Mahony, J. A., McSweeney, P. L. H., and Lucey, J. A. (2006). A model system for studying 
the effects of colloidal calcium phosphate concentration on the rheological properties of cheddar cheese. Journal of Dairy Science, 89(3):892-904.

Podlubny, I. (1999). Fractional Differential Equations. Academic Press.

Reiner, M. (1964). The deborah number. Physics today, 17(1):62.

Sahin, S. and Sumnu, S. G. (2006). Physical Properties of Foods. Springer.

Schiessel, H., Metzler, R., Blumen, A., and Nonnenmacher, T. F. (1995). Generalized viscoelastic models: Their fractional equations with solutions. Journal of Physics A: General Physics, 28(23):6567-6584.

Scott Blair, G. W. (1947). The role of psychophysics in rheology. Journal of Colloid Science, 2(1):21-32.

Scott Blair, G. W. and Coppen, F. M. V. (1940a). 273. the consistency of cheese curd at the pitching point and its bearing on the firmness and quality of the finished cheese. Journal of Dairy Research, 12:44-54.

Scott Blair, G. W. and Coppen, F. M. V. (1940b). Subjective judgments of 'firmness' in elastic and viscous materials. Nature, 145(3672):425-426.

Scott Blair, G. W. and Coppen, F. M. V. (1942a). Psycho-physical significance of the dissipation coefficient of soft materials. Nature, 149(3766):22-23.

Scott Blair, G. W. and Coppen, F. M. V. (1942b). The subjective conception of the firmness of soft materials. American Journal of Psychology, 55:215-229.

Scott Blair, G. W., Veinoglou, B. C., and Caffyn, J. E. (1947). Limitations of the Newtonian time scale in relation to non-equilibrium rheological states and a theory of quasi-properties. Proceedings of the Royal Society of London. Series A, Mathematical and physical sciences, 189(1016):69-87.

Sprakel, J., Lindström, S. B., Kodger, T. E., and Weitz, D. A. (2011). Stress enhancement in the delayed yielding of colloidal gels. Physical Review Letters, 106(24).

Szczesniak, A. S. (1963). Objective measurements of food texture. Journal of Food Science, 28(4):410-420.

Szczesniak, A. S. (1975). Textural characterization of temperature sensitive foods. Journal of Texture Studies, 6(1):139-156. 
Tanner, R. I., Qi, F., and Dai, S.-C. (2008). Bread dough rheology and recoil. I. Rheology. Journal of Non-Newtonian Fluid Mechanics, 148(1-3):33-40.

Van Vliet, T. and Walstra, P. (1994). Water in casein gels; how to get it out or keep it in. Journal of Food Engineering, 22(1-4):75-88.

Visser, F. M. W. (1977). Contribution of enzymes from rennet, starter bacteria and milk to proteolysis and flavour development in gouda cheese. 3. protein breakdown: Analysis of the soluble nitrogen and amino acid nitrogen fractions. Netherlands Milk and Dairy Journal, 31:210-239.

Walstra, P. (1968). Estimating globule-size distribution of oil-in-water emulsions by spectroturbidimetry. Journal of Colloid And Interface Science, 27(3):493-500.

Walstra, P. and van Vliet, T. (1991). Rheological and fracture properties of cheese. Number 268 in Bulletin of the International Dairy Federation. International Dairy Federation.

Winter, H. H. and Mours, M. (1997). Rheology of polymers near liquid-solid transitions. Advances in Polymer Science, 134:164-234.

Yang, X., Rogers, N. R., Berry, T. K., and Foegeding, E. A. (2011). Modeling the rheological properties of cheddar cheese with different fat contents at various temperatures. Journal of Texture Studies, 42(5):331-348.

Yates, M. D. and Drake, M. A. (2007). Texture properties of Gouda cheese. Journal of Sensory Studies, 22(5):493-506.

Zhou, N. and Mulvaney, S. J. (1998). The effect of milk fat, the ratio of casein to water, and temperature on the viscoelastic properties of rennet casein gels. Journal of Dairy Science, 81(10):2561-2571. 
Appendix A. Appendices

Appendix A.1. Table of needed equations 





Appendix A.2. Fractional Maxwell Model Expressions for Springiness and Rubberiness

Appendix A.2.1. Springiness

The equation for the springiness for the FMM is given by

$$
S \approx\left|\frac{-\Delta t_{s}^{\beta-1}}{\mathrm{G} \Gamma(1+\beta)}+\frac{-\Delta t_{s}^{\alpha-1}}{\mathrm{~V} \Gamma(1+\alpha)}\right|,
$$

which can be rewritten as

$$
S \approx \frac{\Delta t_{s}^{\alpha-1}}{\mathbb{V} \Gamma(1+\alpha)}\left|\frac{\mathbb{V}}{\mathbb{G}} \Delta t_{s}^{\beta-\alpha} \frac{\Gamma(1+\alpha)}{\Gamma(1+\beta)}+1\right|
$$

Substituting the expression for $\tau_{c}$ Eq. A.13) gives

$$
S \approx \frac{\Delta t_{s}^{\alpha-1}}{\mathrm{~V} \Gamma(1+\alpha)}\left|\left(\frac{\tau_{c}}{\Delta t_{s}}\right)^{\alpha-\beta} \frac{\Gamma(1+\alpha)}{\Gamma(1+\beta)}+1\right|
$$

which reduces in the limits of $\tau_{c}<<\Delta t_{s}$ and $\tau_{c}>>\Delta t_{s}$ to

$$
\begin{aligned}
& S \approx\left|\frac{-\Delta t_{s}^{\alpha-1}}{\mathbb{V} \Gamma(1+\alpha)}\right|, \text { for } \tau_{c}<<\Delta t_{s} \\
& S \approx\left|\frac{-\Delta t_{s}^{\beta-1}}{\operatorname{G} \Gamma(1+\beta)}\right|, \text { for } \tau_{c}>>\Delta t_{s}
\end{aligned}
$$

\section{Appendix A.2.2. Rubberiness}

The definition for rubberiness is given by Eq. A.20). Substituting the definitions for the compliance of the fractional Maxwell Model for the creep phase, Eq. A.10, and the recovery phase, Eq. A.12, respectively, plus the times for measuring firmness, $t_{f}$, and rubberiness, $t_{r}$, gives

$$
R=1-\left(\frac{\left(t_{r}^{\alpha}-\Delta t_{r}^{\alpha}\right)+\frac{\mathbb{V}}{\mathbb{G}} \frac{\Gamma(1+\alpha)}{\Gamma(1+\beta)}\left(t_{r}^{\beta}-\Delta t_{r}^{\beta}\right)}{t_{f}^{\alpha}+\frac{\mathbb{V}}{\mathbb{G}} \frac{\Gamma(1+\alpha)}{\Gamma(1+\beta)} t_{f}^{\beta}}\right)
$$


which can be rewritten as

$$
R=1-\left(\frac{t_{r}}{t_{f}}\right)^{\alpha}\left(\frac{1-\left(\frac{t_{r}}{t_{f}}\right)^{\alpha}+\frac{\mathbb{V}}{\mathbb{G}} \frac{\Gamma(1+\alpha)}{\Gamma(1+\beta)}\left(t_{r}^{\beta-\alpha}-\frac{\Delta t_{r}^{\beta}}{t_{r}^{\alpha}}\right)}{1+\frac{\mathbb{V}}{\mathbb{G}} \frac{\Gamma(1+\alpha)}{\Gamma(1+\beta)} t_{f}^{\beta-\alpha}}\right)
$$

Substitution of the definition of $\tau_{c}$, A.13 gives

$$
R=1-\left(\frac{t_{r}}{t_{f}}\right)^{\alpha}\left(\frac{1-\left(\frac{t_{r}}{t_{f}}\right)^{\alpha}+\frac{\Gamma(1+\alpha)}{\Gamma(1+\beta)}\left(\left(\frac{\tau_{c}}{t_{r}}\right)^{\alpha-\beta}-\tau_{c}^{\alpha-\beta} \frac{\Delta t_{r}^{\beta}}{t_{r}^{\alpha}}\right)}{1+\frac{\Gamma(1+\alpha)}{\Gamma(1+\beta)}\left(\frac{\tau_{c}}{t_{f}}\right)^{\alpha-\beta}}\right)
$$

When we set $t_{f}=\Delta t_{r}$ we get

$$
R=2-2^{\beta}+\frac{2^{\beta}-2^{\alpha}}{1+\frac{\Gamma(1+\alpha)}{\Gamma(1+\beta)}\left(\frac{\tau_{c}}{t_{f}}\right)^{\alpha-\beta}}
$$

which reduces in the limits $\tau_{c} / t_{f}<<1$ and $\tau_{c} / t_{f}>>1$ to

$$
\begin{aligned}
& R=2-2^{\alpha}, \text { for } \tau_{c} / t_{f}<<1 \text { and } t_{f}=t_{r} \\
& R \approx 2-2^{\beta}, \text { for } \tau_{c} / t_{f}>>1 \text { and } t_{f}=t_{r}
\end{aligned}
$$


Table A.2: Overview of parameter values obtained by fitting either a single-element fractional constitutive model (SB) or the two-element Fractional Maxwell Model (FMM) to small strain linear viscoelastic data and used in subsequent predictions which are indicated by the use of a bold font (SB,FMM). For predictions of $G^{*}(\omega), G(t)$, and $J(t)$ the model parameters are independently extracted from an independent material function, indicated in the column 'Source', which was measured using a new sample from the same batch of cheese. Predictions of the creep compliance in the recovery phase, $J^{-}(t)$ are obtained by fitting either the SB or FMM model to the measured creep compliance of the creep phase, $J^{+}(t)$.

\begin{tabular}{|c|c|c|c|c|c|c|c|c|c|}
\hline Fig. & Function & Model & Source & $\begin{array}{c}\text { Fat } \\
{[w \%]}\end{array}$ & $\begin{array}{c}\mathrm{T} \\
{\left[{ }^{\circ} \mathrm{C}\right]}\end{array}$ & $\begin{array}{c}\mathbb{G} / 10^{5} \\
{\left[\begin{array}{lll}\mathrm{Pas}^{\beta}\end{array}\right]}\end{array}$ & $\begin{array}{c}\beta \\
{[-]}\end{array}$ & $\begin{array}{l}\mathbb{V} / 10^{5} \\
{\left[\mathrm{Pas}^{\alpha}\right]}\end{array}$ & $\begin{array}{c}\alpha \\
{[-]}\end{array}$ \\
\hline \multirow{5}{*}{2} & $G^{*}(\omega)$ & SB & & 0 & 10 & 0.45 & 0.18 & & \\
\hline & & SB & & 0 & 30 & 0.18 & 0.21 & & \\
\hline & & SB & & 30 & 10 & 1.3 & 0.16 & & \\
\hline & & SB & & 30 & 30 & 0.20 & 0.18 & & \\
\hline & & FMM & $G(t)$ & 30 & 10 & 1.3 & 0.16 & 47 & 0.65 \\
\hline \multirow{3}{*}{$3(a)$} & $G(t)$ & SB & $G^{*}(\omega)$ & 0 & 10 & 0.45 & 0.18 & & \\
\hline & & SB & & 30 & 10 & 1.3 & 0.16 & & \\
\hline & & FMM & & 30 & 10 & 1.3 & 0.16 & 47 & 0.65 \\
\hline \multirow{2}{*}{$3(b)$} & $J(t)$ & SB & $G^{*}(\omega)$ & 0 & 10 & 0.45 & 0.18 & & \\
\hline & & SB & & 30 & 10 & 1.7 & 0.15 & & \\
\hline \multirow[t]{2}{*}{4} & $J^{-}(t)$ & SB & $J^{+}(t)$ & 0 & 10 & 0.38 & 0.19 & & \\
\hline & & SB & & 30 & 10 & 1.6 & 0.18 & & \\
\hline \multirow[t]{2}{*}{$5(\mathbf{g}, \mathbf{h})^{\circ}{ }^{\prime} 3^{\prime}$} & $J^{-}(t)$ & SB & $J^{+}(t)$ & 30 & 25 & 0.35 & 0.21 & & \\
\hline & & FMM & & 30 & 25 & 0.34 & 0.13 & 7.6 & 1.0 \\
\hline
\end{tabular}

